\title{
Numerical Implementation of Nonlinear Implicit Iterative Method for Solving Ill-posed Problems
}

\author{
Jianjun Liu \\ PetroChina Pipeline R\&D Center, Langfang, P.R.C \\ Email: kjliujj@petrochina.com.cn
}

Zhe Wang

Research and Innovation Institute Watchdata System Co., Ltd. BeiJing, P.R.C

Email: zhe.wang@watchdata.com

Guoqiang He

Department of Mathematics, Shanghai University, Shanghai, P.R.C

Email: gqhe@staff.shu.edu.cn

Chuangang Kang

Department of Mathematics, Tianjin Polytechnic University, Tianjin, P.R.C

Email: ckang78@sohu.com

\begin{abstract}
Many nonlinear regularization methods may converge to local minima in numerical implementation for the complexity of nonlinear operator. Under some not very strict assumptions, we implement our proposed nonlinear implicit iterative method and have a global convergence results. Using the convexity property of the modified Tikhonov functional, it combines nonlinear implicit iterative method with a gradient method for solving ill-posed problems. Finally we present two numerical results for integral equation and parameter identification.
\end{abstract}

Index Terms-nonlinear, gradient method, regularization, implicit iterative

\section{INTRODUCTION}

This paper is dedicated to the computation of an approximation to a nonlinear operator equation

$$
\mathrm{F}(\mathrm{x})=\mathrm{y} \text {, }
$$

where $\mathrm{F}: \mathrm{D}(\mathrm{F}) \mathrm{X} \rightarrow \mathrm{Y}$ is a nonlinear operator between two real Hilbert spaces $X$ and $Y$. If only noisy data $y^{\delta}$ with

$$
\left\|\mathrm{y}^{\delta}-\mathrm{y}\right\| \leq \delta
$$

are available, then the problem of solving (1) has to be regularized. Nonlinear ill-posed problems are more different to solve than linear. Due to the importance for technical applications, many of the known linear regularization methods have been generalized to nonlinear equations[1,2].

In [3], we extend the implicit iterative method for linear ill-posed operator equations to solve nonlinear illposed problems, named nonlinear implicit iterative

Footnotes: 8-point Times New Roman font;

Manuscript received January 1, 2008; revised June 1, 2008; accepted July 1, 2008

Copyright credit, project number, corresponding author, etc. method. Under some conditions, the error sequence of solutions of the nonlinear implicit iterative method is monotonically decreasing. And with this monotonicity, we prove the convergence of our method for both the exact and perturbed equations. In this paper, we focus on the numerical implementation of nonlinear implicit iterative method. The main part in the numerical implementation is how to minimize the Tikhonov functional in each iteration. When regularization parameter is fixed, how to minimize the Tikhonov functional (3) is a well-posed optimization problem. In principle, all of nonlinear optimization methods can be implied to implement the nonlinear implicit iterative method. But, the convergence may not be hold since the local convergence of some methods and the non-strictly convex of the Tikhonov functional.

$$
\mathrm{J}_{\alpha}(\mathrm{x})=\left\|\mathrm{y}^{\delta}-\mathrm{F}(\mathrm{x})\right\|^{2}+\alpha\left\|\mathrm{x}-\mathrm{x}_{0}\right\|^{2}
$$

In the numerical implementation of nonlinear implicit iterative method, we combine this method with the steepest descent method and develop an iterative algorithm for solving nonlinear ill-posed problems. It maintains the advantage of iteration methods in numerical realization and get a convergence result under some not very strict assumptions. In every iteration, we modify Tikhonov functional by idea of nonlinear implicit iterative method, replacing $\mathrm{x}^{+}$with $\mathrm{x}_{\mathrm{n}}^{\delta}$ :

$$
\mathrm{J}_{\alpha}\left(\mathrm{x}, \mathrm{x}_{\mathrm{n}}^{\delta}\right)=\left\|\mathrm{y}^{\delta}-\mathrm{F}(\mathrm{x})\right\|^{2}+\alpha\left\|\mathrm{x}-\mathrm{x}_{\mathrm{n}}^{\delta}\right\|^{2}
$$

The resulting method will be defined by

$$
\mathrm{x}_{\mathrm{n}, \mathrm{k}+1}^{\delta}=\mathrm{x}_{\mathrm{n}, \mathrm{k}}^{\delta}+\beta_{\mathrm{k}} \nabla \mathrm{J}_{\alpha}\left(\mathrm{x}_{\mathrm{n}, \mathrm{k}}^{\delta}, \mathrm{x}_{\mathrm{n}}^{\delta}\right)
$$

Here, $\beta_{\mathrm{k}}$ denotes a scaling parameter. Since it is based on the interaction of nonlinear implicit iterative method with the gradient method, we feel that 'Implicit Iterative-GRAdient method ' (IIGRA) is an appropriate name for the iteration.

The structure of the paper will be as follows. In section II, it will be shown that the modified Tikhonov 
functional (4) has a convexity property in the neighbourhood of a global minimizer of the functional. A convergence analysis for the steepest descent algorithm for minimizing (4) is given in section III. It is shown that the algorithm converges to a global minimizer of (4) if a starting value for the iteration within the abovementioned neighbourhood is known. Finally, in section IV, we will give two applications. Conclusion is in section $\mathrm{V}$.

\section{RESUlts ON THE CONVEXITY PROPERTY OF MODIFIED TIKHONOV FUNCTIONAL}

Throughout this paper we have the following assumption:

Assumption 1 Suppose there is a ball $\mathrm{B}\left(\mathrm{x}^{+}, \mathrm{r}\right) \subset \mathrm{D}(\mathrm{F})$ with radius $r$ around $x^{+}$and in the ball operator $F$ satisfies:

(i) $\mathrm{F}$ is twice Fréchet differentiable with a continuous second derivative;

(ii) local Lipschitz condition: for $\mathrm{x}_{1}, \mathrm{x}_{2} \in \mathrm{B}\left(\mathrm{x}^{+}, \mathrm{r}\right)$ there holds

$$
\left\|\mathrm{F}^{\prime}\left(\mathrm{x}_{1}\right)-\mathrm{F}^{\prime}\left(\mathrm{x}_{2}\right)\right\| \leq \mathrm{L}\left\|\mathrm{x}_{1}-\mathrm{x}_{2}\right\| ;
$$
holds

(iii) $\exists$ some const $\eta>0$, for all $x_{1}, x_{2} \in B\left(x^{+}, r\right)$ there $\left\|\mathrm{F}\left(\mathrm{x}_{1}\right)-\mathrm{F}\left(\mathrm{x}_{2}\right)-\mathrm{F}^{\prime}\left(\mathrm{x}_{2}\right)\left(\mathrm{x}_{1}-\mathrm{x}_{2}\right)\right\| \leq \eta\left\|\mathrm{x}_{1}-\mathrm{x}_{2}\right\|\left\|\mathrm{F}\left(\mathrm{x}_{1}\right)-\mathrm{F}\left(\mathrm{x}_{2}\right)\right\|$; As a deduction, we can conclude that $\mathrm{F}^{\prime}$ is local bounded in $\mathrm{B}\left(\mathrm{x}^{+}, \mathrm{r}\right)$ from assumption 1 (i), i.e.

$$
\mathrm{M}=\sup \left\{\left\|\mathrm{F}^{\prime}(\mathrm{x}) \mid \mathrm{x} \in \mathrm{B}\left(\mathrm{x}^{+}, \mathrm{r}\right)\right\|\right\}<\infty
$$

Our aim is to use the steepest descent method for minimizing $\mathrm{J}_{\alpha}\left(\mathrm{x}, \mathrm{x}_{\mathrm{n}}^{\delta}\right)$. It is well known that the steepest descent method will converge to (the global) minimizer of a functional, if it is convex. So we will prove that the modified Tikhonov functional has a convexity property in the neighbourhood of a global minimizer of the functional. Since limited by pages, we only give the convergence results without proof in the following, detail seen in [4].

From now on we will set

$$
C_{x}(t)(h, h):=\int_{0}^{1}(1-\tau) F^{\prime \prime}(x+t \tau h)(h, h) d \tau .
$$

By Taylor formula in integral form

$\mathrm{F}(\mathrm{x}+\mathrm{th})=\mathrm{F}(\mathrm{x})+\mathrm{tF}(\mathrm{x}) \mathrm{h}+\int_{0}^{1}(1-\tau) \mathrm{F}^{\prime \prime}(\mathrm{x}+\mathrm{t} \tau \mathrm{h})(\mathrm{h}, \mathrm{h}) \mathrm{d} \tau$, we have

$$
F(x+t h)=F(x)+t F^{\prime}(x) h+t^{2} C_{x}(t)(h, h) .
$$

Let

$$
\varphi_{\alpha, \mathrm{h}}(\mathrm{t})=\mathrm{J}_{\alpha}\left(\mathrm{x}_{\mathrm{n}+1}^{\delta}+\mathrm{th}, \mathrm{x}_{\mathrm{n}}^{\delta}\right), \mathrm{t} \in \mathrm{R}, \mathrm{h} \in \mathrm{X},\|\mathrm{h}\|=1,
$$

using (9) we get

$\mathrm{J}_{\alpha}\left(\mathrm{x}+\mathrm{th}, \mathrm{x}_{\mathrm{n}}^{\delta}\right)=\mathrm{J}_{\alpha}\left(\mathrm{x}, \mathrm{x}_{\mathrm{n}}^{\delta}\right)-2 \mathrm{t}\left(\left\langle\mathrm{y}^{\delta}-\mathrm{F}(\mathrm{x}), \mathrm{F}^{\prime}(\mathrm{x}) \mathrm{h}\right\rangle-\alpha\left\langle\mathrm{x}-\mathrm{x}_{\mathrm{n}}^{\delta},\right\rangle\right)$ $+\mathrm{t}^{2}\left(\left\|\mathrm{~F}^{\prime}(\mathrm{x}) \mathrm{h}\right\|^{2}-2\left\langle\mathrm{y}^{\delta}-\mathrm{F}(\mathrm{x}), \mathrm{C}_{\mathrm{x}}(\mathrm{t})(\mathrm{h}, \mathrm{h})\right\rangle+\alpha\|\mathrm{h}\|^{2}\right)$ $+2 \mathrm{t}^{3}\left\langle\mathrm{~F}^{\prime}(\mathrm{x}) \mathrm{h}, \mathrm{C}_{\mathrm{x}}(\mathrm{t})(\mathrm{h}, \mathrm{h})\right\rangle+\mathrm{t}^{4}\left\|\mathrm{C}_{\mathrm{x}}(\mathrm{t})(\mathrm{h}, \mathrm{h})\right\|^{2}$ Proposition 2 Let $\mathrm{C}_{\mathrm{x}}(\mathrm{t})(\mathrm{h}, \mathrm{h})$ be defined as in (8), and define $g(t): R^{+} \rightarrow Y$ by

$$
g(t):=t^{2} C_{x}(t)(h, h)
$$

Then $\mathrm{g}(\mathrm{t})$ is twice differentiable and the following properties hold:

(i) $\quad \mathrm{g}(\mathrm{t})=\int_{0}^{t}(\mathrm{t}-\tau) \mathrm{F}^{\prime \prime}(\mathrm{x}+\tau \mathrm{h})(\mathrm{h}, \mathrm{h}) \mathrm{d} \tau$.

(ii) $\quad g^{\prime}(\mathrm{t})=\int_{0}^{\mathrm{t}} \mathrm{F}^{\prime \prime}(\mathrm{x}+\tau \mathrm{h})(\mathrm{h}, \mathrm{h}) \mathrm{d} \tau=\mathrm{t} \int_{0}^{1} \mathrm{~F}^{\prime \prime}(\mathrm{x}+\mathrm{t} \tau \mathrm{h})(\mathrm{h}, \mathrm{h}) \mathrm{d} \tau$.

(iii) $\quad \mathrm{g}^{\prime \prime}(\mathrm{t})=\mathrm{F}^{\prime \prime}(\mathrm{x}+\tau \mathrm{h})(\mathrm{h}, \mathrm{h})$

(iv) $\quad\left[\|\mathrm{g}(\mathrm{t})\|^{2}\right]^{\prime \prime}=2\left(\left\langle\mathrm{~g}^{\prime \prime}(\mathrm{t}), \mathrm{g}(\mathrm{t})\right\rangle+\left\|\mathrm{g}^{\prime}(\mathrm{t})\right\|^{2}\right)$.
Proposition 3 Let $C_{x}(t)(h, h)$ be as in $(8)$ and $\hat{C}_{x_{n+1}}^{\delta}(t)(h, h)$ be defined by

$$
\hat{\mathrm{C}}_{\mathrm{x}_{+1}{ }_{\mathrm{s}}}(\mathrm{t})(\mathrm{h}, \mathrm{h}):=\int_{0}^{1} \mathrm{~F}^{\prime \prime}\left(\mathrm{x}_{\mathrm{n}+1}^{\delta}+\mathrm{t} \tau \mathrm{h}\right)(\mathrm{h}, \mathrm{h}) \mathrm{d} \tau,
$$

Then we obtain for the second derivative of $\varphi_{\alpha, \mathrm{h}}(\mathrm{t})$ with $\|\mathrm{h}\|=1$

$$
\begin{aligned}
\varphi_{a, \mathrm{~h}}^{\prime \prime}(\mathrm{t}) & =2\left\|\mathrm{~F}^{\prime}\left(\mathrm{x}_{\mathrm{n}+1}^{\delta}\right) \mathrm{h}\right\|^{2}+2 \alpha+2 \mathrm{t}^{2}\left\|\hat{\mathrm{C}}_{\mathrm{x}_{\mathrm{n}+1}^{\delta}}(\mathrm{t})(\mathrm{h}, \mathrm{h})\right\|^{2} \\
& -2\left\langle\mathrm{y}^{\delta}-\mathrm{F}\left(\mathrm{x}_{\mathrm{n}+1}^{\delta}\right), \mathrm{F}^{\prime \prime}\left(\mathrm{x}_{\mathrm{n}+1}^{\delta}+\mathrm{th}\right)(\mathrm{h}, \mathrm{h})\right\rangle \\
& +\mathrm{t}\left\langle\mathrm{F}^{\prime}\left(\mathrm{x}_{\mathrm{n}+1}^{\delta}\right) \mathrm{h}, 4 \hat{\mathrm{C}}_{\mathrm{x}_{\mathrm{n}+1}}(\mathrm{t})(\mathrm{h}, \mathrm{h})+2 \mathrm{~F}^{\prime \prime}\left(\mathrm{x}_{\mathrm{n}+1}^{\delta}+\mathrm{th}\right)(\mathrm{h}, \mathrm{h})\right\rangle \\
& +2 \mathrm{t}^{2}\left\langle\mathrm{~F}^{\prime \prime}\left(\mathrm{x}_{\mathrm{n}+1}^{\delta}+\mathrm{th}\right)(\mathrm{h}, \mathrm{h}), \hat{\mathrm{C}}_{\mathrm{x}_{\mathrm{n}+1}}^{\delta}(\mathrm{t})(\mathrm{h}, \mathrm{h})\right\rangle .
\end{aligned}
$$

To simplify the notation, we will define

$$
\begin{aligned}
\mathrm{a}(\mathrm{t}, \mathrm{h}):= & 2\left\langle\mathrm{~F}^{\prime \prime}\left(\mathrm{x}_{\mathrm{n}+1}^{\delta}+\mathrm{th}\right)(\mathrm{h}, \mathrm{h}), \hat{\mathrm{C}}_{\mathrm{x}_{\mathrm{n}+1}}^{{ }^{\circ}}(\mathrm{t})(\mathrm{h}, \mathrm{h})\right\rangle \\
& +2\left\|\hat{\mathrm{C}}_{\mathrm{x}_{+1} \mathrm{n}}(\mathrm{t})(\mathrm{h}, \mathrm{h})\right\|^{2}, \\
\mathrm{~b}(\mathrm{t}, \mathrm{h}):= & \left\langle\mathrm{F}^{\prime}\left(\mathrm{x}_{\mathrm{n}+1}^{\delta}\right) \mathrm{h}, 4 \hat{\mathrm{C}}_{\mathrm{x}_{+1}{ }^{\mathrm{n}}}(\mathrm{t})(\mathrm{h}, \mathrm{h})\right. \\
& \left.+2 \mathrm{~F}^{\prime \prime}\left(\mathrm{x}_{\mathrm{n}+1}^{\delta}+\mathrm{th}\right)(\mathrm{h}, \mathrm{h})\right\rangle, \\
\mathrm{c}(\mathrm{t}, \mathrm{h}):= & 2 \alpha-2\left\langle\mathrm{y}^{\delta}-\mathrm{F}\left(\mathrm{x}_{\mathrm{n}+1}^{\delta}\right), \mathrm{F}^{\prime \prime}\left(\mathrm{x}_{\mathrm{n}+1}^{\delta}+\mathrm{th}\right)(\mathrm{h}, \mathrm{h})\right\rangle \\
+ & 2\left\|\mathrm{~F}^{\prime}\left(\mathrm{x}_{\mathrm{n}+1}^{\delta}\right) \mathrm{h}\right\|^{2}
\end{aligned}
$$

and have therefore

$$
\varphi_{\alpha, h}^{\prime \prime}(t)=c(t, h)+t b(t, h)+t^{2} a(t, h)
$$

Proposition 4 Let $a(t, h), b(t, h)$ and $c(t, h)$ be defined as in (13)-(15). If $1-\mathrm{L}\left\|\mathrm{y}^{\delta}-\mathrm{F}\left(\mathrm{x}_{0}^{\delta}\right)\right\| / \alpha>0$, then there must be some $\gamma>0$ such that

$$
\gamma+\mathrm{L}\left\|\mathrm{y}^{\delta}-\mathrm{F}\left(\mathrm{x}_{0}^{\delta}\right)\right\| / \alpha<1
$$

holds. Then $a(t, h), b(t, h)$ and $c(t, h)$ can be estimated independently of $\mathrm{t}, \mathrm{h}$ :

$$
\begin{aligned}
& |\mathrm{a}(\mathrm{t}, \mathrm{h})| \leq 3 \mathrm{~L}^{2} \\
& |\mathrm{~b}(\mathrm{t}, \mathrm{h})| \leq 6 \mathrm{LM} \\
& \mathrm{c}(\mathrm{t}, \mathrm{h})>2 \gamma \alpha,
\end{aligned}
$$

where $\mathrm{L}$ and $\mathrm{M}$ are defined as (6) and (7).

Proof. For a Lipschitz-continuous first Fréchet derivative with (6), the second derivative can be globally estimated using

$$
\left\|\mathrm{F}^{\prime \prime}(\mathrm{x})(\mathrm{h}, \mathrm{h})\right\| \leq \mathrm{L}\|\mathrm{h}\|^{2}
$$

According to the definition of $\mathrm{C}_{\mathrm{x}}(\mathrm{t})(\mathrm{h}, \mathrm{h})$ as in (8) and $\hat{\mathrm{C}}_{\mathrm{X}_{\mathrm{n}+1}}^{\delta}(\mathrm{t})(\mathrm{h}, \mathrm{h})$ as in (12) we have with $\|\mathrm{h}\|=1$

$$
\begin{aligned}
& \left\|\mathrm{C}_{\mathrm{x}_{n+1}}(\mathrm{t})(\mathrm{h}, \mathrm{h})\right\| \leq \int_{0}^{1}(1-\tau)\left\|\mathrm{F}^{\prime \prime}\left(\mathrm{x}_{\mathrm{n}+1}^{\delta}+\mathrm{t} \tau \mathrm{h}\right)(\mathrm{h}, \mathrm{h})\right\| \mathrm{d} \tau \leq \frac{\mathrm{L}}{2}, \\
& \left\|\hat{\mathrm{C}}_{\mathrm{x}_{\mathrm{n}+1}}(\mathrm{t})(\mathrm{h}, \mathrm{h})\right\| \leq \int_{0}^{1}\left\|\mathrm{~F}^{\prime \prime}\left(\mathrm{x}_{\mathrm{n}+1}^{\delta}+\mathrm{t} \tau \mathrm{h}\right)(\mathrm{h}, \mathrm{h}) \quad\right\| \mathrm{d} \tau \leq \mathrm{L} .
\end{aligned}
$$
and thus

$$
\begin{aligned}
|\mathrm{a}(\mathrm{t}, \mathrm{h})| \leq & 2\left\|\mathrm{~F}^{\prime \prime}\left(\mathrm{x}_{\mathrm{n}+1}^{\delta}+\mathrm{th}\right)(\mathrm{h}, \mathrm{h})\right\|\left\|\mathrm{C}_{\mathrm{x}_{\mathrm{n}+1}}(\mathrm{t})(\mathrm{h}, \mathrm{h})\right\| \\
& +2\left\|\hat{\mathrm{C}}_{\mathrm{x}_{+1} \mathrm{n}}^{{ }^{\mathrm{n}}}(\mathrm{t})(\mathrm{h}, \mathrm{h})\right\|^{2} \leq 3 \mathrm{~L}^{2} .
\end{aligned}
$$

Similarly

$$
\begin{aligned}
|\mathrm{b}(\mathrm{t}, \mathrm{h})| \leq & \left\|\mathrm{F}^{\prime}\left(\mathrm{x}_{\mathrm{n}+1}^{\delta}\right)\right\|\left(4\left\|\hat{\mathrm{C}}_{\mathrm{x}_{\mathrm{n}+1}}(\mathrm{t})(\mathrm{h}, \mathrm{h})\right\|\right. \\
& \left.+2\left\|\mathrm{~F}^{\prime \prime}\left(\mathrm{x}_{\mathrm{n}+1}^{\delta}+\mathrm{th}\right)(\mathrm{h}, \mathrm{h})\right\|\right)
\end{aligned}
$$$$
\leq 6 \mathrm{~L}\left\|\mathrm{~F}^{\prime}\left(\mathrm{x}_{+1}^{\delta \mathrm{n}}\right) \quad\right\| \leq 6 \mathrm{LM} \text {. }
$$

$\mathrm{c}(\mathrm{t}, \mathrm{h})$ can be estimated as follows:

$\mathrm{c}(\mathrm{t}, \mathrm{h})-2 \gamma \alpha=2\left\|\mathrm{~F}^{\prime}\left(\mathrm{x}_{\mathrm{n}+1}^{\delta}\right) \mathrm{h}\right\|^{2}+2 \alpha$

$$
\begin{aligned}
& -2\left\langle\mathrm{y}^{\delta}-\mathrm{F}\left(\mathrm{x}_{\mathrm{n}+1}^{\delta}\right), \mathrm{F}^{\prime \prime}\left(\mathrm{x}_{\mathrm{n}+1}^{\delta}+\mathrm{th}\right)(\mathrm{h}, \mathrm{h})\right\rangle-2 \gamma \alpha \\
& \geq 2 \alpha-2 \mathrm{~L}\left\|\mathrm{y}^{\delta}-\mathrm{F}\left(\mathrm{x}_{\mathrm{n}+1}^{\delta}\right)\right\|-2 \gamma \alpha \\
& \geq 2 \alpha-2 \mathrm{~L}\left\|\mathrm{y}^{\delta}-\mathrm{F}\left(\mathrm{x}_{0}^{\delta}\right)\right\|-2 \gamma \alpha \\
& =2 \alpha\left(1-\mathrm{L}\left\|\mathrm{y}^{\delta}-\mathrm{F}\left(\mathrm{x}_{0}^{\delta}\right)\right\| / \alpha-\gamma\right)>0 .
\end{aligned}
$$

Theorem 5 Under assumption (18) and $\|\mathrm{h}\|=1$, the functional $\varphi_{\alpha, \mathrm{h}}(\mathrm{t})$ is a convex function for all

$$
|\mathrm{t}| \leq \frac{1}{\mathrm{~L}(1+\sqrt{2})} \min \left\{\sqrt{\frac{2 \kappa \alpha}{3}}, \frac{2 \kappa \alpha}{3 \mathrm{M}}\right\}:=\mathrm{r}(\alpha),
$$

where $\kappa=1-\mathrm{L}\left\|\mathrm{y}^{\delta}-\mathrm{F}\left(\mathrm{x}_{0}^{\delta}\right)\right\| / \alpha-\gamma$. Particularly, it holds

$$
\varphi_{\alpha, \mathrm{h}}^{\prime \prime}(\mathrm{t}) \geq 2 \gamma \alpha
$$


for $|t| \leq r(\alpha)$.

Proof. We have

$$
\varphi_{a, h}^{\prime \prime}(t)=c(t, h)+t b(t, h)+t^{2} a(t, h)
$$

We have to consider two cases:

(1) Let $t>0$. Then

$\varphi_{a, h}^{\prime \prime}(t) \geq c(t, h)-t|b(t, h)|-t^{2}|a(t, h)|$ for $b(t, h) \leq 0$

$\varphi_{a, h}^{\prime \prime}(\mathrm{t}) \geq \mathrm{c}(\mathrm{t}, \mathrm{h})-\mathrm{t}^{2}|\mathrm{a}(\mathrm{t}, \mathrm{h})|$

for $b(t, h)>0$

(2) Let $t \leq 0$. Then we have

$$
\varphi_{\alpha, \mathrm{h}}^{\prime \prime}(\mathrm{t}) \geq \mathrm{c}(\mathrm{t}, \mathrm{h})-\mathrm{t}^{2}|\mathrm{a}(\mathrm{t}, \mathrm{h})| \quad \text { for } \mathrm{b}(\mathrm{t}, \mathrm{h}) \leq 0
$$$$
\varphi_{a, h}^{\prime \prime \prime}(t) \geq c(t, h)-t|b(t, h)|-t^{2}|a(t, h)| \text { for } b(t, h)>0
$$

Thus it is sufficient to consider the first case only. Setting

$$
\kappa=1-\mathrm{L}\left\|\mathrm{y}^{\delta}-\mathrm{F}\left(\mathrm{x}_{0}^{\delta}\right)\right\| / \alpha-\gamma
$$

then $\kappa>0$ because of (18).

From (29) it follows, with (19) and (25), that

$$
\varphi_{\alpha, \mathrm{h}}^{\prime \prime}(\mathrm{t})-2 \gamma \alpha \geq 2 \kappa \alpha-3 \mathrm{~L}^{2} \mathrm{t}^{2}=: \mathrm{p}_{1}(\mathrm{t}) \text {. }
$$

$\mathrm{p}_{1}(\mathrm{t})$ has the zeros

$$
\mathrm{t}_{1,2}= \pm \sqrt{\frac{2 \kappa}{3 \mathrm{~L}^{2}}} \sqrt{\alpha}
$$

and because $p_{1}(0)>0$, it holds that

for $|\mathrm{t}| \leq\left|\mathrm{t}_{1,2}\right|$.

$$
\varphi_{\alpha, \mathrm{h}}^{\prime \prime}(\mathrm{t}) \geq 2 \gamma \alpha
$$

From (28) it follows, with (19) and (20) that

$$
\varphi_{\alpha, \mathrm{h}}^{\prime \prime}(\mathrm{t})--2 \gamma \alpha \geq 2 \kappa \alpha-3 \mathrm{LMt}-3 \mathrm{~L}^{2} \mathrm{t}^{2}=: \mathrm{p}_{2}(\mathrm{t}) \text {. }
$$

and $\mathrm{p}_{2}(\mathrm{t})$ has the zeros

$$
\mathrm{t}_{1,2}=\frac{1}{\mathrm{~L}}\left[-\mathrm{M} \pm \sqrt{\mathrm{M}^{2}+\frac{2 \kappa \alpha}{3}}\right] .
$$

Now let $\mathrm{t}_{\min }=\min \left\{\left|\mathrm{t}_{1}\right|,\left|\mathrm{t}_{2}\right|\right\}$. Then

$$
\begin{aligned}
t_{\min } & =\frac{1}{L}\left[-M+\sqrt{M^{2}+\frac{2 \kappa \alpha}{3}}\right] \\
& =\frac{2}{3 L} \frac{\kappa \alpha}{M+\sqrt{M^{2}+\frac{2}{3} \kappa \alpha}} \\
& \geq \frac{1}{L(1+\sqrt{2})} \begin{cases}\frac{2 \kappa \alpha}{3 M} & \text { if } \frac{2}{3} \kappa \alpha \leq M^{2} \\
\sqrt{\frac{2}{3} \kappa \alpha} & \text { if } \frac{2}{3} \kappa \alpha>M^{2}\end{cases}
\end{aligned}
$$

Combining (31), (33) we have shown that $\varphi_{a, \mathrm{~h}}^{\prime \prime}(\mathrm{t}) \geq 2 \gamma \alpha$ for

$$
\begin{aligned}
|\mathrm{t}| & \leq \frac{1}{\mathrm{~L}} \min \left\{\sqrt{\frac{2 \kappa \alpha}{3}}, \frac{1}{1+\sqrt{2}} \sqrt{\frac{2 \kappa \alpha}{3}}, \frac{2 \kappa \alpha}{3 \mathrm{M}(1+\sqrt{2})}\right\} \\
& =\frac{1}{\mathrm{~L}(1+\sqrt{2})} \min \left\{\sqrt{\frac{2 \kappa \alpha}{3}}, \frac{2 \kappa \alpha}{3 \mathrm{M}}\right\} .
\end{aligned}
$$

\section{A SteEPESt Descent Method}

The steepest descent method is a widely used iteration method for minimizing a functional. Although it is sometimes slow to converge, it seldom fails to converge to a minimum of the functional. The method is defined by

$$
\mathrm{x}_{\mathrm{n}, \mathrm{k}+1}^{\delta}=\mathrm{x}_{\mathrm{n}, \mathrm{k}}^{\delta}+\beta_{\mathrm{k}} \nabla \mathrm{J}_{\alpha}\left(\mathrm{x}_{\mathrm{n}, \mathrm{k}}^{\delta}, \mathrm{x}_{\mathrm{n}}^{\delta}\right) \quad \mathrm{k}=0,1,2, \ldots \ldots,
$$

where $\nabla \mathrm{J}_{\alpha}\left(\mathrm{x}_{\mathrm{n}, \mathrm{k}}^{\delta}, \mathrm{x}_{\mathrm{n}}^{\delta}\right)$ denotes the direction of steepest descent of $\mathrm{J}_{\alpha}\left(\mathrm{x}, \mathrm{x}_{\mathrm{n}}^{\delta}\right)$ at point $\mathrm{x}_{\mathrm{n}, \mathrm{k}}^{\delta}$, and $\beta_{\mathrm{k}} \in \mathrm{R}^{+}$is a step size or scaling parameter which has to be chosen according to

$$
\beta_{\mathrm{k}}=\arg \min _{\beta \geq 0}\left\{\mathrm{~J}_{\alpha}\left(\mathrm{x}_{\mathrm{n}, \mathrm{k}}^{\delta}+\beta \nabla \mathrm{J}_{\alpha}\left(\mathrm{x}_{\mathrm{n}, \mathrm{k}}^{\delta}, \mathrm{x}_{\mathrm{n}}^{\delta}\right), \mathrm{x}_{\mathrm{n}}^{\delta}\right)\right\} .
$$

According to (10) we find

$$
\nabla \mathrm{J}_{\alpha}\left(\mathrm{x}_{\mathrm{n}, \mathrm{k}}^{\delta}, \mathrm{x}_{\mathrm{n}}^{\delta}\right)=-2\left(\mathrm{~F}^{\prime}\left(\mathrm{x}_{\mathrm{n}, \mathrm{k}}^{\delta}\right) *\left(\mathrm{y}^{\delta}-\mathrm{F}\left(\mathrm{x}_{\mathrm{n}, \mathrm{k}}^{\delta}\right)\right)-\alpha\left(\mathrm{x}_{\mathrm{n}, \mathrm{k}}^{\delta}-\mathrm{x}_{\mathrm{n}}^{\delta}\right)\right) .
$$

For the following, we might use the notation
$\mathrm{K}_{\mathrm{r}(\alpha)}\left(\mathrm{x}_{\mathrm{n}+1}^{\delta}\right):=\left\{\mathrm{x} \in \mathrm{X} \mid \mathrm{x}=\mathrm{x}_{\mathrm{n}+1}^{\delta}+\mathrm{th}, \mathrm{t} \in \mathrm{R}, \mathrm{h} \in \mathrm{X}, \|\right.$ th $\left.\| \leq \mathrm{r}(\alpha)\right\}$.

Define

$\mathrm{h}_{\mathrm{k}}:=\mathrm{x}_{\mathrm{n}+1}^{\delta}-\mathrm{x}_{\mathrm{n}, \mathrm{k}}^{\delta}$

and functions $\varphi_{1}, \varphi_{2}$ by

$$
\begin{aligned}
& \varphi_{1}(\mathrm{t})=\mathrm{J}_{\alpha}\left(\mathrm{x}_{\mathrm{n}, \mathrm{k}}^{\delta}+\mathrm{th}, \mathrm{x}_{\mathrm{n}}^{\delta}\right) \\
& \varphi_{2}(\mathrm{t})=\mathrm{J}_{\alpha}\left(\mathrm{x}_{\mathrm{n}, \mathrm{k}}^{\delta}-\mathrm{th}, \mathrm{x}_{\mathrm{n}}^{\delta}\right)
\end{aligned}
$$

$\varphi_{1}$ and $\varphi_{2}$ can be rewritten as

$$
\begin{aligned}
\varphi_{1}(\mathrm{t}) & =\varphi_{1}(0)+2\left\langle\nabla \mathrm{J}_{\alpha}\left(\mathrm{x}_{\mathrm{n}, \mathrm{k}}^{\delta}, \mathrm{x}_{\mathrm{n}}^{\delta}\right), \mathrm{h}_{\mathrm{k}}\right\rangle \mathrm{t}+\mathrm{c}_{1}\left(\mathrm{t}, \mathrm{h}_{\mathrm{k}}\right) \mathrm{t}^{2} \\
& +\mathrm{b}_{1}\left(\mathrm{t}, \mathrm{h}_{\mathrm{k}}\right) \mathrm{t}^{3}+\mathrm{a}_{1}\left(\mathrm{t}, \mathrm{h}_{\mathrm{k}}\right) \mathrm{t}^{{ }^{4}} \\
\varphi_{2}(\mathrm{t}) & =\varphi_{2}(0)-2\left\langle\nabla \mathrm{J}_{\alpha}\left(\mathrm{x}_{\mathrm{n}+1}^{\delta}, \mathrm{x}_{\mathrm{n}}^{\delta}\right), \mathrm{h}_{\mathrm{k}}\right\rangle \mathrm{t}+\mathrm{c}_{2}\left(\mathrm{t}, \mathrm{h}_{\mathrm{k}}\right) \mathrm{t}^{2} \\
& +\mathrm{b}_{2}\left(\mathrm{t}, \mathrm{h}_{\mathrm{k}}\right) \mathrm{t}^{3}+\mathrm{a}_{2}\left(\mathrm{t}, \mathrm{h}_{\mathrm{k}}\right) \mathrm{t}^{4}
\end{aligned}
$$

where the coefficients $c_{1}\left(t, h_{k}\right), b_{1}\left(t, h_{k}\right), a_{1}\left(t, h_{k}\right), c_{2}\left(t, h_{k}\right)$, $b_{2}\left(t, h_{k}\right), a_{2}\left(t, h_{k}\right)$ can be determined as in (10), eg.

$\left.\mathrm{c}_{1}\left(\mathrm{t}, \mathrm{h}_{\mathrm{k}}\right)=\left\|\mathrm{F}^{\prime}\left(\mathrm{x}_{\mathrm{n}, \mathrm{k}}^{\delta}\right) \mathrm{h}_{\mathrm{k}}\right\|^{2}-2\left\langle\mathrm{y}^{\delta}-\mathrm{F}\left(\mathrm{x}_{\mathrm{n}, \mathrm{k}}^{\delta}\right), \mathrm{C}_{\mathrm{x}_{\mathrm{n}, \mathrm{k}}}(\mathrm{t}) \mathrm{h}_{\mathrm{k}}, \mathrm{h}_{\mathrm{k}}\right\rangle\right\rangle$, $\left.\mathrm{b}_{1}\left(\mathrm{t}, \mathrm{h}_{\mathrm{k}}\right)=2\left\langle\mathrm{~F}^{\prime}\left(\mathrm{x}_{\mathrm{n}, \mathrm{k}}^{\delta}\right) \mathrm{h}_{\mathrm{k}}, \mathrm{C}_{\mathrm{x}_{\mathrm{n}, \mathrm{k}}}(\mathrm{t}) \mathrm{h}_{\mathrm{k}}, \mathrm{h}_{\mathrm{k}}\right)\right\rangle$

$\left.\mathrm{a}_{1}\left(\mathrm{t}, \mathrm{h}_{\mathrm{k}}\right)=\| \mathrm{C}_{\mathrm{x}_{\mathrm{n}, \mathrm{k}}}(\mathrm{t}) \mathrm{h}_{\mathrm{k}}, \mathrm{h}_{\mathrm{k}}\right) \|^{2}$

Proposition 6 Let the assumption of theorem 5 hold, and $\operatorname{let} \varphi_{1}(\mathrm{t})$ be defined as in (40) and $\mathrm{x}_{\mathrm{n}, \mathrm{k}}^{\delta} \in \mathrm{K}_{\mathrm{r}(\alpha)}\left(\mathrm{x}_{\mathrm{n}+1}^{\delta}\right)$. Then we have

$$
\begin{aligned}
& \varphi_{1}{ }^{\prime}(t)<0 \text { for } t \in[0,1] \\
& \varphi_{1}{ }^{\prime}(t)=0 \text { for } t=1
\end{aligned}
$$

and

$$
\varphi_{1}^{\prime \prime}(\mathrm{t}) \geq 2 \gamma \alpha\left\|\mathrm{h}_{\mathrm{k}}\right\|^{2}, \mathrm{t} \in[0,1] .
$$

Proposition 7 Assume that $\mathrm{x}_{\mathrm{n}, \mathrm{k}}^{\delta} \in \mathrm{K}_{\mathrm{r}(\alpha)}\left(\mathrm{x}_{\mathrm{n}+1}^{\delta}\right)$. Then an interval $\mathrm{I}=\left(0, \beta_{0}\right]$,

$$
\left.\left.\beta_{0}=\frac{\left\langle-\nabla \mathrm{J}_{\alpha}\left(\mathrm{x}_{\mathrm{n}, \mathrm{k}}^{\delta}, \mathrm{x}_{\mathrm{n}}^{\delta}\right), \mathrm{h}_{\mathrm{k}}\right\rangle}{\left\|\nabla \mathrm{J}_{\alpha}\left(\mathrm{x}_{\mathrm{n}, \mathrm{k}}^{\delta}, \mathrm{x}_{\mathrm{n}}^{\delta}\right)\right\|^{2}}, \mathrm{k}_{\mathrm{k}}^{\delta} \quad, \mathrm{x}_{\mathrm{n}}^{\delta}\right) \|^{2}\right) .
$$

exists such that the iterate $\mathrm{x}_{\mathrm{n}, \mathrm{k}+1}^{\delta}=\mathrm{x}_{\mathrm{n}, \mathrm{k}}^{\delta}-\beta_{\mathrm{k}} \nabla \mathrm{J}_{\alpha}\left(\mathrm{x}_{\mathrm{n}, \mathrm{k}}^{\delta}, \mathrm{x}_{\mathrm{n}}^{\delta}\right)$ is closer to $\mathrm{x}_{\mathrm{n}+1}^{\delta}$ than $\mathrm{x}_{\mathrm{n}, \mathrm{k}}^{\delta}$ for $\beta_{\mathrm{k}} \in \mathrm{I}$ :

$$
\left\|\mathrm{x}_{\mathrm{n}+1}^{\delta}-\mathrm{x}_{\mathrm{n}, \mathrm{k}+1}^{\delta}\right\| \leq\left\|\mathrm{x}_{\mathrm{n}+1}^{\delta}-\mathrm{x}_{\mathrm{n}, \mathrm{k}}^{\delta}\right\| .
$$

In particular, $\mathrm{x}_{\mathrm{n}, \mathrm{k}+1}^{\delta} \in \mathrm{K}_{\mathrm{r}(\alpha)}\left(\mathrm{x}_{\mathrm{n}+1}^{\delta}\right)$.

Proposition 8 Let the assumptions of theorem 3 hold, and $x_{n, j}^{\delta}, j=0, \ldots, k$ be the steepest descent iterates for minimizing $\mathrm{J}_{\alpha}\left(\mathrm{x}, \mathrm{x}_{\mathrm{n}}^{\delta}\right)$, and $\mathrm{h}_{\mathrm{k}}$ be defined as in (37) Moreover assume $x_{n, j}^{\delta} \in K_{r(\alpha)}\left(x_{n+1}^{\delta}\right) j=0, \ldots k$. Then

$$
\left\langle-\nabla \mathrm{J}_{\alpha}\left(\mathrm{x}_{\mathrm{n}, \mathrm{k}}^{\delta}, \mathrm{x}_{\mathrm{n}}^{\delta}\right), \mathrm{h}_{\mathrm{k}}\right\rangle \geq \gamma \alpha\left\|\mathrm{h}_{\mathrm{k}}\right\|^{2}
$$

Define

$$
\left.\lambda=\frac{4 \gamma \alpha}{4 \mathrm{M}^{2}+4 \alpha+4 \mathrm{~L}\left\|\mathrm{y}^{\delta}-\mathrm{F}\left(\mathrm{x}_{0}^{\delta}\right)\right\|+4 \mathrm{LM}+\mathrm{L}^{2}}+\mathrm{L}^{2}\right) .
$$

Proposition 9 Let the assumptions of theorem 5 hold, and $x_{n, j}^{\delta}, j=0, \ldots, k$ be the steepest descent iterates for minimizing $\mathrm{J}_{\alpha}\left(\mathrm{x}, \mathrm{x}_{\mathrm{n}}^{\delta}\right)$. If $\mathrm{x}_{\mathrm{n}, \mathrm{j}}^{\delta} \in \mathrm{K}_{\mathrm{r}(\alpha)}\left(\mathrm{x}_{\mathrm{n}+1}^{\delta}\right), \mathrm{j}=0, \ldots \mathrm{k}$, and the scaling parameter $\beta_{\mathrm{k}}$ is chosen such that

$\left.\beta_{\mathrm{k}} \leq \min \left\{\frac{\gamma \alpha}{\left\|\nabla \mathrm{J}_{\alpha}\left(\mathrm{x}_{\mathrm{n}, \mathrm{k}}^{\delta}, \mathrm{x}_{\mathrm{n}}^{\delta}\right)\right\|^{2}} \square \delta{ }_{\mathrm{n}} \quad\right) \|^{2}\right)$, $\left.\left.\left.\lambda \frac{\mathrm{J}_{\alpha}\left(\mathbf{x}_{\mathrm{n}, \mathrm{k}}^{\delta}, \mathbf{x}_{\mathrm{n}}^{\delta}\right)-\theta_{\min \mathrm{k}}}{\left\|\nabla \mathbf{J}_{\alpha}\left(\mathbf{x}_{\mathrm{n}, \mathrm{k}}^{\delta}, \mathbf{x}_{\mathrm{n}}^{\delta}\right)\right\|^{2}, \mathrm{k}} \quad, \mathbf{x}_{\mathrm{n}}^{\delta}\right) \|^{2}\right)\right\}$

holds, then the new iterate $x_{n, k+1}^{\delta}$ is closer to $x_{n+1}^{\delta}$ than $x_{n, k}^{\delta}$. Here,

$$
\theta_{\min , \mathrm{k}}=\min \left\{\mathrm{J}_{\alpha}\left(\mathrm{x}_{\mathrm{n}, \mathrm{k}}^{\delta} \mathrm{t} \nabla \mathrm{J}_{\alpha}\left(\mathrm{x}_{\mathrm{n}, \mathrm{k}}^{\delta}, \mathrm{x}_{\mathrm{n}}^{\delta}\right), \mathrm{x}_{\mathrm{n}}^{\delta}\right): \mathrm{t} \in \mathrm{R}^{+}\right\} .
$$

In particular, $\mathrm{x}_{\mathrm{n}, \mathrm{k}+1}^{\delta} \in \mathrm{K}_{\mathrm{r}(\alpha)}\left(\mathrm{x}_{\mathrm{n}+1}^{\delta}\right)$.

Proof. Let $\varphi_{1}(\mathrm{t}), \varphi_{2}(\mathrm{t})$ be defined as in (38)-(41). We will first estimate $\left\|h_{k}\right\|$ from below by $\varphi_{1}(0)-\varphi_{1}(1)$. Keeping in mind that $\varphi_{1}(\mathrm{t})=\varphi_{1}(1-\mathrm{t})$ and $\nabla \mathrm{J}_{\alpha}\left(\mathrm{x}_{\mathrm{n}+1}^{\delta}, \mathrm{x}_{\mathrm{n}}^{\delta}\right)=0$ hold, we get from (41) 
On setting

$$
\begin{aligned}
\varphi_{1}(0)-\varphi_{1}(1) & =\varphi_{2}(1)-\varphi_{2}(0) \\
& =c_{2}\left(t, h_{k}\right)+b_{2}\left(t, h_{k}\right)+a_{2}\left(t, h_{k}\right) .
\end{aligned}
$$

$\mathrm{C}_{\mathrm{k}, \mathrm{x}_{\mathrm{n}+1}^{\delta}}^{\delta}(\mathrm{t})\left(\mathrm{h}_{\mathrm{k}}, \mathrm{h}_{\mathrm{k}}\right)=\int_{0}^{1}(1-\tau) \mathrm{F}^{\prime \prime}\left(\mathrm{x}_{\mathrm{n}+1}^{\delta}+\mathrm{t} \tau \mathrm{h}_{\mathrm{k}}\right)\left(\mathrm{h}_{\mathrm{k}}, \mathrm{h}_{\mathrm{k}}\right) \mathrm{d} \tau,(47)$ the coefficients in (46) can be determined from

$\mathrm{c}_{2}\left(\mathrm{t}, \mathrm{h}_{\mathrm{k}}\right)=\left\|\mathrm{F}^{\prime}\left(\mathrm{x}_{\mathrm{n}+1}^{\delta}\right) \mathrm{h}_{\mathrm{k}}\right\|^{2}-2\left\langle\mathrm{y}^{\delta}-\mathrm{F}\left(\mathrm{x}_{\mathrm{n}+1}^{\delta}\right), \mathrm{C}_{\mathrm{k}, \mathrm{x}_{\mathrm{n}+1}^{\delta}}(\mathrm{t})\left(\mathrm{h}_{\mathrm{k}}, \mathrm{h}_{\mathrm{k}}\right)\right\rangle$, $\mathrm{b}_{2}\left(\mathrm{t}, \mathrm{h}_{\mathrm{k}}\right)=2\left\langle\mathrm{~F}^{\prime}\left(\mathrm{x}_{\mathrm{n}+1}^{\delta}\right) \mathrm{h}_{\mathrm{k}}, \mathrm{C}_{\mathrm{k}, \mathrm{x}_{\mathrm{n}+1}^{\circ}}(\mathrm{t})\left(\mathrm{h}_{\mathrm{k}}, \mathrm{h}_{\mathrm{k}}\right)\right\rangle$

$\mathrm{a}_{2}\left(\mathrm{t}, \mathrm{h}_{\mathrm{k}}\right)=\left\|\mathrm{C}_{\mathrm{k}, \mathrm{x}_{\mathrm{n}+1}}(\mathrm{t})\left(\mathrm{h}_{\mathrm{k}}, \mathrm{h}_{\mathrm{k}}\right)\right\|^{2}$

Using

$$
\left\|\mathrm{C}_{\mathrm{k}, \mathrm{x}_{\mathrm{n}+1}}^{\delta}(\mathrm{t})\left(\mathrm{h}_{\mathrm{k}}, \mathrm{h}_{\mathrm{k}}\right)\right\| \leq \mathrm{L} \int_{0}^{1}(1-\tau) \mathrm{d} \tau\left\|\mathrm{h}_{\mathrm{k}}\right\|^{2}=\frac{\mathrm{L}}{2}\left\|\mathrm{~h}_{\mathrm{k}}\right\|^{2}
$$

we obtain

$$
\begin{aligned}
& \left|\mathrm{c}_{2}\left(\mathrm{t}, \mathrm{h}_{\mathrm{k}}\right)\right| \leq\left(\mathrm{M}^{2}+\alpha+\mathrm{L}\left\|\mathrm{y}^{\delta}-\mathrm{F}\left(\mathrm{x}_{0}^{\delta}\right)\right\|\right) \quad\left\|\mathrm{h}_{\mathrm{k}}\right\|^{2} \\
& =\overline{\mathrm{c}_{2}} \quad\left\|\mathrm{~h}_{\mathrm{k}}\right\|^{2} \text {, } \\
& \left|\mathrm{b}_{2}\left(\mathrm{t}, \mathrm{h}_{\mathrm{k}}\right)\right| \leq \mathrm{LM}\left\|\mathrm{h}_{\mathrm{k}}\right\|^{3}=: \overline{\mathrm{b}_{2}}\left\|\mathrm{~h}_{\mathrm{k}}\right\|^{3} \text {, } \\
& \left|\mathrm{a}_{2}\left(\mathrm{t}, \mathrm{h}_{\mathrm{k}}\right)\right| \leq \frac{\mathrm{L}^{2}}{4}\left\|\mathrm{~h}_{\mathrm{k}}\right\|^{4}=\overline{\mathrm{a}_{2}}\left\|\mathrm{~h}_{\mathrm{k}}\right\|^{4} \text {. }
\end{aligned}
$$

Altogether this yields

$$
\varphi_{1}(0)-\varphi_{1}(1) \leq \overline{\mathrm{c}_{2}} \quad\left\|\mathrm{~h}_{\mathrm{k}}\right\|^{2}+\overline{\mathrm{b}_{2}} \quad\left\|\mathrm{~h}_{\mathrm{k}}\right\|^{3}+\overline{\mathrm{a}_{2}} \quad\left\|\mathrm{~h}_{\mathrm{k}}\right\|^{4}
$$

The minimal value of $J_{\alpha}\left(x_{n+1}^{\delta}, x_{n}^{\delta}\right)=\varphi_{1}(1)$ is usually unknown, but $\theta_{\text {min,k }}$, defined in (45), can be computable. In the case of $\left\|\mathrm{h}_{\mathrm{k}}\right\| \leq 1$ we find

$\varphi_{1}(0)-\theta_{\min , \mathrm{k}} \leq \varphi_{1}(0)-\varphi_{1}(1)$

$$
\begin{aligned}
& \leq\left(\mathrm{M}^{2}+\alpha+\mathrm{L}\left\|\mathrm{y}^{\delta}-\mathrm{F}\left(\mathrm{x}_{0}^{\delta}\right)\right\|\right)\left\|\mathrm{h}_{\mathrm{k}}\right\|^{2} \\
& +\mathrm{LM}\left\|\mathrm{h}_{\mathrm{k}}\right\|^{3}+\frac{\mathrm{L}^{2}}{4}\left\|\mathrm{~h}_{\mathrm{k}}\right\|^{4} \\
& \leq\left(\mathrm{M}^{2}+\alpha+\mathrm{L}\left\|\mathrm{y}^{\delta}-\mathrm{F}\left(\mathrm{x}_{0}^{\delta}\right)\right\|\right. \\
& \left.+\mathrm{LM}+\frac{\mathrm{L}^{2}}{4}\right)\left\|\mathrm{h}_{\mathrm{k}}\right\|^{2} .
\end{aligned}
$$

Thus it follows by using () for $\left\|\mathrm{h}_{\mathrm{k}}\right\| \leq 1$ that

$$
\begin{aligned}
\left\langle-\nabla \mathrm{J}_{\alpha}\left(\mathrm{x}_{\mathrm{n}, \mathrm{k}}^{\delta}, \mathrm{x}_{\mathrm{n}}^{\delta}, \mathrm{h}_{\mathrm{k}}\right)\right\rangle & \geq \lambda\left(\varphi_{1}(0)-\theta_{\min , \mathrm{k}}\right) \\
& =\lambda\left(\mathrm{J}_{\alpha}\left(\mathrm{x}_{\mathrm{n}, \mathrm{k}}^{\delta}, \mathrm{x}_{\mathrm{n}}^{\delta}\right)-\theta_{\min , \mathrm{k}}\right) .
\end{aligned}
$$

In the case of $\left\|h_{k}\right\| \geq 1$ we get from ()

$$
\left\langle-\nabla \mathrm{J}_{\alpha}\left(\mathrm{x}_{\mathrm{n}, \mathrm{k}}^{\delta}, \mathrm{x}_{\mathrm{n}}^{\delta}, \mathrm{h}_{\mathrm{k}}\right)\right\rangle \geq \gamma \alpha \text {. }
$$

Inserting the above estimates for $\left\langle-\nabla \mathrm{J}_{\alpha}\left(\mathrm{x}_{\mathrm{n}, \mathrm{k}}^{\delta}, \mathrm{x}_{\mathrm{n}}^{\delta}, \mathrm{h}_{\mathrm{k}}\right)\right\rangle$ in (42) shows that $\beta_{\mathrm{k}} \leq \beta_{0}$, and, by proposition, the new iterate is therefore closer to $x_{n+1}^{\delta}$ than the old one.

Let

$$
\begin{aligned}
\kappa_{1} & :=\operatorname{Lr}(\alpha)+\mathrm{M}, \\
\mathrm{r}^{\delta} & \left.\left.:=\frac{\left\|\mathrm{y}^{\delta}-\mathrm{F}\left(\mathrm{x}_{0}^{\delta}\right)\right\|}{\sqrt{\alpha}}, \alpha\right)\right), \\
\kappa_{3}^{2} & :=\alpha^{2}\left(\mathrm{r}^{2}(\alpha)+\left(\mathrm{r}^{\delta}\right)^{2}\right), \\
\kappa_{2} & :=\left\|\mathrm{y}^{\delta}-\mathrm{F}\left(\mathrm{x}_{0}^{\delta}\right)\right\|^{2}+\operatorname{Mr}(\alpha)+\frac{\mathrm{L}}{2} \mathrm{r}(\alpha)^{2} .
\end{aligned}
$$

Theorem 10 Let the assumptions of theorem 5 hold, $\mathrm{K}_{\mathrm{r}(\alpha)}\left(\mathrm{x}_{\mathrm{n}+1}^{\delta}\right)$ be defined as in (36), $\mathrm{x}_{\mathrm{n}, 0}^{\delta}=\mathrm{x}_{\mathrm{n}}^{\delta} \in \mathrm{K}_{\mathrm{r}(\alpha)}\left(\mathrm{x}_{\mathrm{n}+1}^{\delta}\right)$, and $\left\{\mathrm{x}_{\mathrm{n}, \mathrm{k}}^{\delta}\right\}_{\mathrm{k} \in \mathrm{N}}$ be the iterates of steepest descent for the modified Tikhonov functional with $\beta_{\mathrm{k}}$ chosen according to proposition 9 . Then, there exists a constant

$$
\begin{aligned}
& \mathrm{K}:=2 \kappa_{1}^{2}+2 \mathrm{~L} \kappa_{2}+2 \alpha+6 \kappa_{1} \kappa^{*}+3 \mathrm{~L}^{2} \kappa_{3}, \\
& \kappa^{*}:=2\left(\kappa_{1} \kappa_{2}+\kappa_{3}\right),
\end{aligned}
$$

such that the second derivative of

$$
\theta_{\mathrm{k}}(\mathrm{t}):=\mathrm{J}_{\alpha}\left(\mathrm{x}_{\mathrm{n}, \mathrm{k}}^{\delta}-\mathrm{t} \nabla \mathrm{J}_{\alpha}\left(\mathrm{x}_{\mathrm{n}, \mathrm{k}}^{\delta}, \mathrm{x}_{\mathrm{n}}^{\delta}\right), \mathrm{x}_{\mathrm{n}}^{\delta}\right) \quad \mathrm{t} \in[0,1]
$$

$$
\left|\theta^{\prime \prime}(\mathrm{t})\right| \leq \mathrm{K}\left\|\nabla \mathrm{J}_{\alpha}\left(\mathrm{x}_{\mathrm{n}, \mathrm{k}}^{\delta}, \mathrm{x}_{\mathrm{n}}^{\delta}\right)\right\|^{2} .
$$

Proof. According to the choice $\beta_{\mathrm{k}}$ all iterates are in $\mathrm{K}_{\mathrm{r}(\alpha)}\left(\mathrm{x}_{\mathrm{n}+1}^{\delta}\right)$. The definition of $\nabla \mathrm{J}_{\alpha}\left(\mathrm{x}_{\mathrm{n}, \mathrm{k}}^{\delta}, \mathrm{x}_{\mathrm{n}}^{\delta}\right)$ shows that $\left\|\nabla \mathrm{J}_{\alpha}\left(\mathrm{x}_{\mathrm{n}, \mathrm{k}}^{\delta}, \mathrm{x}_{\mathrm{n}}^{\delta}\right)\right\|$ is bounded in $\mathrm{K}_{\mathrm{r}(\alpha)}\left(\mathrm{x}_{\mathrm{n}+1}^{\delta}\right)$ by a constant $\kappa^{*}$ defined in (53), we have

$$
\begin{aligned}
& \left\|\nabla \mathrm{J}_{\alpha}\left(\mathrm{x}_{\mathrm{n}, \mathrm{k}}^{\delta}, \mathrm{x}_{\mathrm{n}}^{\delta}\right)\right\| \\
& \leq 2\left(\left\|\mathrm{~F}^{\prime}\left(\mathrm{x}_{\mathrm{n}, \mathrm{k}}^{\delta}\right)\right\|\left\|\mathrm{y}^{\delta}-\mathrm{F}^{\prime}\left(\mathrm{x}_{\mathrm{n}, \mathrm{k}}^{\delta}\right)\right\|+\alpha\left\|\mathrm{x}_{\mathrm{n}, \mathrm{k}}^{\delta}-\mathrm{x}_{\mathrm{n}}^{\delta}\right\|\right) .
\end{aligned}
$$
and

$$
\left\|F^{\prime}\left(x_{n, k}^{\delta}\right)\right\| \leq\left\|F^{\prime}\left(x_{n, k}^{\delta}\right)-F^{\prime}\left(x_{n+1}^{\delta}\right)\right\|+\left\|F^{\prime}\left(x_{n+1}^{\delta}\right)\right\|
$$$$
\leq \mathrm{L}\left\|\mathrm{x}_{+1}^{\delta \mathrm{n}}-\mathrm{x}_{\mathrm{n}, \mathrm{k}}^{\delta}\right\| \leq \mathrm{M}+\mathrm{Lr}(\alpha)=\kappa_{1} \text {, }
$$

$\left\|\mathrm{y}^{\delta}-\mathrm{F}^{\prime}\left(\mathrm{x}_{\mathrm{n}, \mathrm{k}}^{\delta}\right)\right\| \leq\left\|\mathrm{y}^{\delta}-\mathrm{F}^{\prime}\left(\mathrm{x}_{\mathrm{n}+1}^{\delta}\right)\right\|\left\|\mathrm{F}^{\prime}\left(\mathrm{x}_{\mathrm{n}+1}^{\delta}\right)-\mathrm{F}^{\prime}\left(\mathrm{x}_{\mathrm{n}, \mathrm{k}}^{\delta}\right)\right\|$ $\leq\left\|\mathrm{y}^{\delta}-\mathrm{F}^{\prime}\left(\mathrm{x}_{0}^{\delta}\right)\right\|+\left\|\mathrm{F}^{\prime}\left(\mathrm{x}_{\mathrm{n}+1}^{\delta}\right)\right\|\left\|\mathrm{x}_{\mathrm{n}+1}^{\delta}-\mathrm{x}_{\mathrm{n}, \mathrm{k}}^{\delta}\right\|$ $+\frac{\mathrm{L}}{2}\left\|\mathrm{x}_{\mathrm{n}+1}^{\delta}-\mathrm{x}_{\mathrm{n}, \mathrm{k}}^{\delta}\right\|^{2}$

$$
\begin{gathered}
\leq\left\|\mathrm{y}^{\delta}-\mathrm{F}^{\prime}\left(\mathrm{x}_{0}^{\delta}\right)\right\|+\mathrm{Mr}(\alpha)+\frac{\mathrm{L}}{2} \mathrm{r}(\alpha)^{2}=\kappa_{2}, \\
\alpha^{2}\left\|\mathrm{x}_{\mathrm{n}, \mathrm{k}}^{\delta}-\mathrm{x}_{\mathrm{n}}^{\delta}\right\|^{2} \\
\leq \alpha^{2}\left(\left\|\mathrm{x}_{\mathrm{n}, \mathrm{k}}^{\delta}-\mathrm{x}_{\mathrm{n}+1}^{\delta}\right\|^{2}+\left\|\mathrm{x}_{\mathrm{n}+1}^{\delta}-\mathrm{x}_{\mathrm{n}}^{\delta}\right\|^{2}\right) \\
\leq \alpha^{2}\left(\mathrm{r}(\alpha)^{2}+\left(\mathrm{r}^{\delta}\right)^{2}\right)=\kappa_{3}^{2} .
\end{gathered}
$$

Thus

$$
\left\|\nabla \mathbf{J}_{\alpha}\left(\mathbf{x}_{\mathrm{n}, \mathrm{k}}^{\delta}, \mathrm{x}_{\mathrm{n}}^{\delta}\right)\right\| \leq \boldsymbol{\kappa}^{*} .
$$

$\left\|\nabla \mathrm{J}_{\alpha}\left(\mathbf{x}_{\mathrm{n}, \mathrm{k}}^{\delta}, \mathrm{x}_{\mathrm{n}}^{\delta}\right)\right\| \leq \mathrm{\kappa}^{*}$

holds for all $k$. Defining $C_{k, x_{n+1}}(t)\left(h_{k}, h_{k}\right)$ as in (47) with $\mathrm{h}_{\mathrm{k}}$ replaced by $-\nabla \mathrm{J}_{\alpha}\left(\mathrm{x}_{\mathrm{n}, \mathrm{k}}^{\delta}, \mathrm{x}_{\mathrm{n}}^{\delta}\right)$ and setting

$\overline{\mathrm{C}}_{\mathrm{k}, \mathrm{x}_{\mathrm{n}+1}^{\delta}}(\mathrm{t})\left(-\nabla \mathrm{J}_{\alpha}\left(\mathrm{x}_{\mathrm{n}, \mathrm{k}}^{\delta}, \mathrm{x}_{\mathrm{n}}^{\delta}\right),-\nabla \mathrm{J}_{\alpha}\left(\mathrm{x}_{\mathrm{n}, \mathrm{k}}^{\delta}, \mathrm{x}_{\mathrm{n}}^{\delta}\right)\right)$

$=\int_{0}^{1} F^{\prime \prime}\left(x_{n, k}^{\delta}-t \tau \nabla J_{\alpha}\left(x_{n, k}^{\delta}, x_{n}^{\delta}\right)\right)\left(-\nabla J_{\alpha}\left(x_{n, k}^{\delta}, x_{n}^{\delta}\right),-\nabla J_{\alpha}\left(x_{n, k}^{\delta}, x_{n}^{\delta}\right)\right) d \tau$ we find

$\theta_{\mathrm{k}}^{\prime \prime}(\mathrm{t})$

$=2\left\|\mathrm{~F}^{\prime}\left(\mathrm{x}_{\mathrm{n}, \mathrm{k}}^{\delta}\right) \nabla \mathrm{J}_{\alpha}\left(\mathrm{x}_{\mathrm{n}, \mathrm{k}}^{\delta}, \mathrm{x}_{\mathrm{n}}^{\delta}\right)\right\|-2\left\langle\mathrm{y}^{\delta}-\mathrm{F}\left(\mathrm{x}_{\mathrm{n}, \mathrm{k}}^{\delta}\right)\right.$,

$\left.\mathrm{F}^{\prime \prime}\left(\mathrm{x}_{\mathrm{n}, \mathrm{k}}^{\delta}-\mathrm{t} \nabla \mathrm{J}_{\alpha}\left(\mathrm{x}_{\mathrm{n}, \mathrm{k}}^{\delta}, \mathrm{x}_{\mathrm{n}}^{\delta}\right)\right)\left(-\nabla \mathrm{J}_{\alpha}\left(\mathrm{x}_{\mathrm{n}, \mathrm{k}}^{\delta}, \mathrm{x}_{\mathrm{n}}^{\delta}\right),-\nabla \mathrm{J}_{\alpha}\left(\mathrm{x}_{\mathrm{n}, \mathrm{k}}^{\delta}, \mathrm{x}_{\mathrm{n}}^{\delta}\right)\right)\right\rangle$

$+2 \alpha\left\|\nabla \mathbf{J}_{\alpha}\left(\mathbf{x}_{\mathrm{n}, \mathrm{k}}^{\delta}, \mathrm{x}_{\mathrm{n}}^{\delta}\right)\right\|^{2}$

$-4 \mathrm{t}\left\langle\mathrm{F}^{\prime}\left(\mathrm{x}_{\mathrm{n}, \mathrm{k}}^{\delta}\right) \nabla \mathrm{J}_{\alpha}\left(\mathrm{x}_{\mathrm{n}, \mathrm{k}}^{\delta}, \mathrm{x}_{\mathrm{n}}^{\delta}\right), \overline{\mathrm{C}} \underset{\mathrm{k}, \mathrm{x}_{\mathrm{n}+1}}{\delta}(\mathrm{t})\left(-\nabla \mathrm{J}_{\alpha}\left(\mathrm{x}_{\mathrm{n}, \mathrm{k}}^{\delta}, \mathrm{x}_{\mathrm{n}}^{\delta}\right)\right.\right.$,

$\left.\left.-\nabla \mathrm{J}_{\alpha}\left(\mathrm{x}_{\mathrm{n}, \mathrm{k}}^{\delta}, \mathrm{x}_{\mathrm{n}}^{\delta}\right)\right)\right\rangle$

$-2 \mathrm{t}\left\langle\mathrm{F}^{\prime}\left(\mathrm{x}_{\mathrm{n}, \mathrm{k}}^{\delta}\right) \nabla \mathrm{J}_{\alpha}\left(\mathrm{x}_{\mathrm{n}, \mathrm{k}}^{\delta}, \mathrm{x}_{\mathrm{n}}^{\delta}\right), \mathrm{F}^{\prime \prime}\left(\mathrm{x}_{\mathrm{n}, \mathrm{k}}^{\delta} \mathrm{t} \nabla \mathrm{J}_{\alpha}\left(\mathrm{x}_{\mathrm{n}, \mathrm{k}}^{\delta}, \mathrm{x}_{\mathrm{n}}^{\delta}\right)\right)\right.$

- $\left.\left(-\nabla \mathrm{J}_{\alpha}\left(\mathrm{x}_{\mathrm{n}, \mathrm{k}}^{\delta}, \mathrm{x}_{\mathrm{n}}^{\delta}\right),-\nabla \mathrm{J}_{\alpha}\left(\mathrm{x}_{\mathrm{n}, \mathrm{k}}^{\delta}, \mathrm{x}_{\mathrm{n}}^{\delta}\right)\right)\right\rangle$

$+2 \mathrm{t}^{2}\left\langle\mathrm{~F}^{\prime \prime}\left(\mathrm{x}_{\mathrm{n}, \mathrm{k}}^{\delta}-\mathrm{t} \nabla \mathrm{J}_{\alpha}\left(\mathrm{x}_{\mathrm{n}, \mathrm{k}}^{\delta}, \mathrm{x}_{\mathrm{n}}^{\delta}\right)\right)\left(-\nabla \mathrm{J}_{\alpha}\left(\mathrm{x}_{\mathrm{n}, \mathrm{k}}^{\delta}, \mathrm{x}_{\mathrm{n}}^{\delta}\right),-\nabla \mathrm{J}_{\alpha}\left(\mathrm{x}_{\mathrm{n}, \mathrm{k}}^{\delta}, \mathrm{x}_{\mathrm{n}}^{\delta}\right)\right)\right.$,

$\left.\mathrm{C}_{\mathrm{k}, \mathrm{x}_{\mathrm{n}+1}}(\mathrm{t})\left(-\nabla \mathrm{J}_{\alpha}\left(\mathrm{x}_{\mathrm{n}, \mathrm{k}}^{\delta}, \mathrm{x}_{\mathrm{n}}^{\delta}\right),-\nabla \mathrm{J}_{\alpha}\left(\mathrm{x}_{\mathrm{n}, \mathrm{k}}^{\delta}, \mathrm{x}_{\mathrm{n}}^{\delta}\right)\right)\right\rangle$

$+2 \mathrm{t}^{2}\left\|\overline{\mathrm{C}} \underset{\mathrm{k}, \mathrm{x}_{\mathrm{n}+1}^{\delta}}{\mathrm{s}}(\mathrm{t})\left(-\nabla \mathrm{J}_{\alpha}\left(\mathrm{x}_{\mathrm{n}, \mathrm{k}}^{\delta}, \mathrm{x}_{\mathrm{n}}^{\delta}\right),-\nabla \mathrm{J}_{\alpha}\left(\mathrm{x}_{\mathrm{n}, \mathrm{k}}^{\delta}, \mathrm{x}_{\mathrm{n}}^{\delta}\right)\right)\right\|^{2}$.

The norm of $\mathrm{C}_{\mathrm{k}, \mathrm{x}_{\mathrm{n}+1}{ }^{\circ}}(\mathrm{t})\left(-\nabla \mathrm{J}_{\alpha}\left(\mathrm{x}_{\mathrm{n}, \mathrm{k}}^{\delta}, \mathrm{x}_{\mathrm{n}}^{\delta}\right),-\nabla \mathrm{J}_{\alpha}\left(\mathrm{x}_{\mathrm{n}, \mathrm{k}}^{\delta}, \mathrm{x}_{\mathrm{n}}^{\delta}\right)\right)$ and $\overline{\mathrm{C}}{ }_{\mathrm{k}, \mathrm{x}_{\mathrm{n}+1}}(\mathrm{t})\left(-\nabla \mathrm{J}_{\alpha}\left(\mathrm{x}_{\mathrm{n}, \mathrm{k}}^{\delta}, \mathrm{x}_{\mathrm{n}}^{\delta}\right),-\nabla \mathrm{J}_{\alpha}\left(\mathrm{x}_{\mathrm{n}, \mathrm{k}}^{\delta}, \mathrm{x}_{\mathrm{n}}^{\delta}\right)\right)$ can be estimated similarly $\mathrm{C}_{\mathrm{k}, \mathrm{x}^{\circ+1}}(\mathrm{t})\left(\mathrm{h}_{\mathrm{k}}, \mathrm{h}_{\mathrm{k}}\right)$ :

$$
\begin{aligned}
& \left\|\mathrm{C}_{\mathrm{k}, \mathrm{x}_{\mathrm{n}+1}}(\mathrm{t})\left(-\nabla \mathrm{J}_{\alpha}\left(\mathrm{x}_{\mathrm{n}, \mathrm{k}}^{\delta}, \mathrm{x}_{\mathrm{n}}^{\delta}\right),-\nabla \mathrm{J}_{\alpha}\left(\mathrm{x}_{\mathrm{n}, \mathrm{k}}^{\delta}, \mathrm{x}_{\mathrm{n}}^{\delta}\right)\right)\right\| \\
& \leq \frac{\mathrm{L}}{2}\left\|\nabla \mathrm{J}_{\alpha}\left(\mathrm{x}_{\mathrm{n}, \mathrm{k}}^{\delta}, \mathrm{x}_{\mathrm{n}}^{\delta}\right)\right\|^{2} . \\
& \left\|\overline{\mathrm{C}} \underset{\mathrm{k}, \mathrm{x}_{\mathrm{n}+1}}{ }(\mathrm{t})\left(-\nabla \mathrm{J}_{\alpha}\left(\mathrm{x}_{\mathrm{n}, \mathrm{k}}^{\delta}, \mathrm{x}_{\mathrm{n}}^{\delta}\right),-\nabla \mathrm{J}_{\alpha}\left(\mathrm{x}_{\mathrm{n}, \mathrm{k}}^{\delta}, \mathrm{x}_{\mathrm{n}}^{\delta}\right)\right)\right\| \\
& \leq \mathrm{L}\left\|\nabla \mathrm{J}_{\alpha}\left(\mathrm{x}_{\mathrm{n}, \mathrm{k}}^{\delta}, \mathrm{x}_{\mathrm{n}}^{\delta}\right)\right\|^{2} .
\end{aligned}
$$

Using the above-given estimates, we can finally estimate $\left|\theta_{\mathrm{k}}^{\prime \prime}(\mathrm{t})\right|$ for $\mathrm{t} \in[0,1]$ by

$$
\begin{aligned}
\left|\theta_{\mathrm{k}}^{\prime \prime}(\mathrm{t})\right| & \leq\left(2 \kappa_{1}^{2}+2 \mathrm{~L} \kappa_{2}+2 \alpha\right)\left\|\nabla \mathrm{J}_{\alpha}\left(\mathrm{x}_{\mathrm{n}, \mathrm{k}}^{\delta}, \mathrm{x}_{\mathrm{n}}^{\delta}\right)\right\|^{2} \\
& +6 \mathrm{t} \kappa_{1} \mathrm{~L}\left\|\nabla \mathrm{J}_{\alpha}\left(\mathrm{x}_{\mathrm{n}, \mathrm{k}}^{\delta}, \mathrm{x}_{\mathrm{n}}^{\delta}\right)\right\|^{3} \\
& +3 \mathrm{t}^{2} \mathrm{~L}^{2}\left\|\nabla \mathrm{J}_{\alpha}\left(\mathrm{x}_{\mathrm{n}, \mathrm{k}}^{\delta}, \mathrm{x}_{\mathrm{n}}^{\delta}\right)\right\|^{4} \\
& \leq\left(2 \kappa_{1}^{2}+2 \mathrm{~L} \kappa_{2}+2 \alpha+6 \kappa_{1} \kappa^{*} \mathrm{~L}+3 \mathrm{~L}^{2}\left(\kappa^{*}\right)^{2}\right) \\
& \cdot\left\|\nabla \mathrm{J}_{\alpha}\left(\mathrm{x}_{\mathrm{n}, \mathrm{k}}^{\delta}, \mathrm{x}_{\mathrm{n}}^{\delta}\right)\right\|^{2} \\
& =\mathrm{K}\left\|\nabla \mathbf{J}_{\alpha}\left(\mathrm{x}_{\mathrm{n}, \mathrm{k}}^{\delta}, \mathrm{x}_{\mathrm{n}}^{\delta}\right)\right\|^{2} .
\end{aligned}
$$


Now all ingredients for the final convergence proof of the steepest descent method have been collected:

Theorem 11 Let the assumptions of theorem 5 hold, $\mathrm{K}_{\mathrm{r}(\alpha)}\left(\mathrm{x}_{\mathrm{n}+1}^{\delta}\right)$ be defined as in $(36), \mathrm{x}_{\mathrm{n}, 0}^{\delta}=\mathrm{x}_{\mathrm{n}}^{\delta} \in \mathrm{K}_{\mathrm{r}(\alpha)}\left(\mathrm{x}_{\mathrm{n}+1}^{\delta}\right)$ and $\left\{\mathrm{x}_{\mathrm{n}, \mathrm{k}}^{\delta}\right\}_{\mathrm{k} \in \mathrm{N}}$ be the iterates of steepest descent for the modified Tikhonov functional with $\beta_{\mathrm{k}}$ chosen such that proposition 4 and

$$
\beta_{\mathrm{k}} \leq \min \left\{\frac{1}{\mathrm{~K}}, 1\right\},
$$

where $\mathrm{K}$ is defined as in (52). Then $\left\{\mathrm{x}_{\mathrm{n}, \mathrm{k}}^{\delta}\right\}_{\mathrm{k} \in \mathrm{N}}$ converges to a global minimizer of $\mathrm{J}_{\alpha}\left(\mathrm{x}, \mathrm{x}_{\mathrm{n}}^{\delta}\right)$ :

$$
\lim _{k \rightarrow \infty} x_{n, k}^{\delta}=x_{n+1}^{\delta}=\arg \min _{x} J_{\alpha}\left(x, x_{n}^{\delta}\right)
$$

Proof. According to the choice $\beta_{\mathrm{k}}$ and $\mathrm{x}_{\mathrm{n}, 0}^{\delta} \in \mathrm{K}_{\mathrm{r}(\alpha)}\left(\mathrm{x}_{\mathrm{n}+1}^{\delta}\right)$, we have

$$
\mathrm{x}_{\mathrm{n}, \mathrm{k}}^{\delta} \in \mathrm{K}_{\mathrm{r}(\alpha)}\left(\mathrm{x}_{\mathrm{n}+1}^{\delta}\right) \mathrm{k} \in \mathrm{N} \text {. }
$$

The sequence $\left\{\mathrm{x}_{\mathrm{n}, \mathrm{k}}^{\delta}\right\}$ is monotonously decreasing and bounded from below, thus there exists $J_{a}^{0}$ s.t $J_{\alpha}\left(x_{n, k}^{\delta}, x_{n}^{\delta}\right) \downarrow J_{a}^{0}$ for $k \rightarrow \infty$. First we will show that $\beta_{\mathrm{k}}$ are bounded from below $\beta, \beta_{\mathrm{k}} \geq \beta$ for all $\mathrm{k}$.

By the definition (33), we have

$$
\theta_{\mathrm{k}}(\mathrm{t})-\theta_{\mathrm{k}}(0)=-\mathrm{t}\left\|\nabla \mathrm{J}_{\alpha}\left(\mathrm{x}_{\mathrm{n}, \mathrm{k}}^{\delta}, \mathrm{x}_{\mathrm{n}}^{\delta}\right)\right\|^{2}+\frac{\mathrm{t}^{2}}{2} \theta_{\mathrm{k}}^{\prime \prime}(\overline{\mathrm{t}}) \quad 0 \leq \overline{\mathrm{t}} \leq \mathrm{t} \text {. }
$$

According to theorem, $\left|\theta_{\mathrm{k}}^{\prime \prime}(\mathrm{t})\right| \leq \mathrm{K}\left\|\nabla \mathbf{J}_{\alpha}\left(\mathbf{x}_{\mathrm{n}, \mathrm{k}}^{\delta}, \mathbf{x}_{\mathrm{n}}^{\delta}\right)\right\|^{2}$. Without loss of generality we can assume $\mathrm{K} \geq 1$. It follows for $0<\mathrm{t} \leq 1$

$$
\begin{aligned}
\theta_{\mathrm{k}}(\mathrm{t})-\theta_{\mathrm{k}}(0) & \leq-\mathrm{t}\left\|\nabla \mathbf{J}_{\alpha}\left(\mathrm{x}_{\mathrm{n}, \mathrm{k}}^{\delta}, \mathrm{x}_{\mathrm{n}}^{\delta}\right)\right\|^{2} \\
& +\frac{\mathrm{t}^{2}}{2} \mathrm{~K}\left\|\nabla \mathbf{J}_{\alpha}\left(\mathbf{x}_{\mathrm{n}, \mathrm{k}}^{\delta}, \mathrm{x}_{\mathrm{n}}^{\delta}\right)\right\|^{2} \\
& =\left(-\mathrm{t}+\frac{\mathrm{t}^{2}}{2} \mathrm{~K}\right)\left\|\nabla \mathbf{J}_{\alpha}\left(\mathrm{x}_{\mathrm{n}, \mathrm{k}}^{\delta}, \mathrm{x}_{\mathrm{n}}^{\delta}\right)\right\|^{2} .
\end{aligned}
$$

If we set esp. $\mathrm{t}=\frac{1}{\mathrm{~K}} \leq 1$, then

$\theta_{\mathrm{k}}\left(\frac{1}{\mathrm{~K}}\right)-\theta_{\mathrm{k}}(0) \leq-\frac{1}{2 \mathrm{~K}}\left\|\nabla \mathrm{J}_{\alpha}\left(\mathrm{x}_{\mathrm{n}, \mathrm{k}}^{\delta}, \mathrm{x}_{\mathrm{n}}^{\delta}\right)\right\|^{2}<0$.

With the definition of $\theta_{\min , \mathrm{k}}$ in (), we find

$\frac{1}{2 \mathrm{~K}}\left\|\nabla \mathbf{J}_{\alpha}\left(\mathbf{x}_{\mathrm{n}, \mathrm{k}}^{\delta}, \mathbf{x}_{\mathrm{n}}^{\delta}\right)\right\|^{2} \leq \theta_{\mathrm{k}}(0)-\theta_{\mathrm{k}}\left(\frac{1}{\mathrm{~K}}\right) \leq \theta_{\mathrm{k}}(0)-\theta_{\min , \mathrm{k}}$

i.e.

$$
\left.\left.\frac{\theta_{\mathrm{k}}(0)-\theta_{\min , \mathrm{k}}}{\left\|\nabla \mathbf{J}_{\alpha}\left(\mathbf{x}_{\mathrm{n}, \mathrm{k}}^{\delta}, \mathbf{x}_{\mathrm{n}}^{\delta}\right)\right\|^{2}} \square \delta_{\mathrm{n}} \quad\right) \|^{2}\right) \geq \frac{1}{2 \mathrm{~K}} .
$$

and from (58) follows

$$
\left.\left.\frac{1}{\left\|\nabla \mathbf{J}_{\alpha}\left(\mathbf{x}_{\mathrm{n}, \mathrm{k}}^{\delta}, \mathbf{x}_{\mathrm{n}}^{\delta}\right)\right\|} \square_{\delta \mathrm{n}} \quad\right) \|\right) \geq \frac{1}{\kappa^{*}} .
$$

Inserting (62), (63) in (60) yields

$$
\beta_{k} \geq \min \left\{\frac{\gamma \alpha}{\kappa^{*}}, \frac{\lambda}{2 \mathrm{~K}}, \frac{1}{\mathrm{~K}}, 1\right\}=: \beta .
$$

Next, we show $\nabla \mathrm{J}_{\alpha}\left(\mathrm{x}_{\mathrm{n}, \mathrm{k}}^{\delta}, \mathrm{x}_{\mathrm{n}}^{\delta}\right) \rightarrow 0$ for $\mathrm{k} \rightarrow 0$ by contradiction. Let us suppose $\nabla \mathrm{J}_{\alpha}\left(\mathrm{x}_{\mathrm{n}, \mathrm{k}}^{\delta}, \mathrm{x}_{\mathrm{n}}^{\delta}\right)$ does not converge to zero. Then there exists $\varepsilon_{0}>0$ s.t. for every $\mathrm{N}_{0} \in \mathrm{N}$ exists $\mathrm{k}>\mathrm{N}_{0}$ with

$$
\left\|\nabla \mathrm{J}_{\alpha}\left(\mathbf{x}_{\mathrm{n}, \mathrm{k}}^{\delta}, \mathbf{x}_{\mathrm{n}}^{\delta}\right)\right\| \geq \varepsilon_{0} .
$$

Because $\mathrm{J}_{\alpha}\left(\mathrm{x}_{\mathrm{n}, \mathrm{k}}^{\delta}, \mathrm{x}_{\mathrm{n}}^{\delta}\right)=\theta_{\mathrm{k}}(0)$ converge monotonously from above to $J_{a}^{0}$, we can moreover assume that

$$
\left|\theta_{\mathrm{k}}(0)-\mathbf{J}_{\mathrm{a}}^{0}\right| \leq \frac{\beta}{4} \varepsilon_{0}^{2} \leq \frac{\beta_{\mathrm{k}}}{4} \varepsilon_{0}^{2} .
$$

holds for $\mathrm{k}$ large enough. Setting $\mathrm{t}=\beta_{\mathrm{k}} \leq \frac{1}{\mathrm{~K}}$, we get

$$
\begin{aligned}
\theta_{\mathrm{k}}\left(\beta_{\mathrm{k}}\right)-\theta_{\mathrm{k}}(0) & \leq \beta_{\mathrm{k}}\left(-1+\frac{\beta_{\mathrm{k}} \mathrm{K}}{2}\right)\left\|\nabla \mathrm{J}_{\alpha}\left(\mathrm{x}_{\mathrm{n}, \mathrm{k}}^{\delta}, \mathrm{x}_{\mathrm{n}}^{\delta}\right)\right\|^{2} \\
& \leq-\frac{\beta_{\mathrm{k}}}{2}\left\|\nabla \mathbf{J}_{\alpha}\left(\mathrm{x}_{\mathrm{n}, \mathrm{k}}^{\delta}, \mathrm{x}_{\mathrm{n}}^{\delta}\right)\right\|^{2} \leq-\frac{\beta_{\mathrm{k}}}{2} \varepsilon_{0}^{2}
\end{aligned}
$$

or

$$
\begin{aligned}
\mathrm{J}_{\alpha}\left(\mathrm{x}_{\mathrm{n}, \mathrm{k}+1}^{\delta}, \mathrm{x}_{\mathrm{n}}^{\delta}\right)-\mathbf{J}_{\mathrm{a}}^{0} & =\theta_{\mathrm{k}}\left(\beta_{\mathrm{k}}\right)-\mathbf{J}_{\mathrm{a}}^{0} \\
& \leq \theta_{\mathrm{k}}(0)-\mathbf{J}_{\mathrm{a}}^{0}-\frac{\beta_{\mathrm{k}}}{2} \varepsilon_{0}^{2} \\
& \leq \frac{\beta_{\mathrm{k}}}{4} \varepsilon_{0}^{2}-\frac{\beta_{\mathrm{k}}}{2} \varepsilon_{0}^{2}=-\frac{\beta_{\mathrm{k}}}{4} \varepsilon_{0}^{2}<0,
\end{aligned}
$$

which is a contradiction to $\nabla \mathrm{J}_{\alpha}\left(\mathrm{x}_{\mathrm{n}, \mathrm{k}}^{\delta}, \mathrm{x}_{\mathrm{n}}^{\delta}\right) \downarrow \mathrm{J}_{\mathrm{a}}^{0}$. As a consequence, $\nabla \mathrm{J}_{\alpha}\left(\mathrm{x}_{\mathrm{n}, \mathrm{k}}^{\delta}, \mathrm{x}_{\mathrm{n}}^{\delta}\right)$ converges to zero. Now by the definition of $h_{k}$ in (37), we finally get by using (43)

$\gamma \alpha\left\|\mathrm{h}_{\mathrm{k}}\right\|^{2} \leq\left\langle-\nabla \mathrm{J}_{\alpha}\left(\mathrm{x}_{\mathrm{n}, \mathrm{k}}^{\delta}, \mathrm{x}_{\mathrm{n}}^{\delta}\right), \mathrm{h}_{\mathrm{k}}\right\rangle$

$$
\leq\left\|\nabla \mathrm{J}_{\alpha}\left(\mathrm{x}_{\mathrm{n}, \mathrm{k}}^{\delta}, \mathrm{x}_{\mathrm{n}}^{\delta}\right)\right\|\left\|\mathrm{h}_{\mathrm{k}}\right\| \rightarrow 0,
$$

and

$\mathrm{x}_{\mathrm{n}, \mathrm{k}}^{\delta} \rightarrow \mathrm{x}_{\mathrm{n}+1}^{\delta}$ for $\left\|\mathrm{h}_{\mathrm{k}}\right\| \rightarrow 0$.

\section{The IIGRA ALgORITHM}

Theorem 12 For the nonlinear implicit iterative method, so long as $x_{0}^{\delta}$ and $\alpha$ is chosen by

(i) $\left.\left\|x_{0}^{\delta}-x^{+}\right\|<\frac{(2 \tau-2) \alpha_{0}^{2}-2 \tau \alpha_{0}-\tau}{2 \tau\left(\frac{L}{\sqrt{\alpha}}+\eta+\frac{\eta}{\tau}\right) \alpha_{0}^{2}}{ }_{0}^{2}\right)$;

(ii) $1-\mathrm{L}\left\|\mathrm{y}^{\delta}-\mathrm{F}\left(\mathrm{x}_{0}^{\delta}\right)\right\|>\gamma>0$;

(iii) $\alpha \geq \min \left\{\alpha_{0}^{2} \mathrm{M}^{2}, \sqrt{\frac{3}{2 \kappa}} \mathrm{L}(1+\sqrt{2})\left\|\mathrm{y} \delta-\mathrm{F}\left(\mathrm{x}_{0}^{\delta}\right)\right\|\right.$,

$$
\left.\left(\frac{3(1+\sqrt{2}) \mathrm{LM}}{2 \kappa}\left\|\mathrm{y}^{\delta}-\mathrm{F}\left(\mathrm{x}_{0}^{\delta}\right)\right\|\right)_{2}^{\frac{3}{2}}\right\} \text {; }
$$

(iv) $\alpha_{0}>\frac{\tau+\sqrt{3 \tau^{2}-2 \tau}}{2 \tau-2}$,

then, the minima $\mathrm{x}_{\mathrm{n}+1}^{\delta}$ of every functional $\mathrm{J}_{\alpha}\left(\mathrm{x}, \mathrm{x}_{\mathrm{n}}^{\delta}\right)$, $\mathrm{n}=0,1, \ldots$ can be given by steepest descent method. The steepest descent method is convergent as $\mathrm{x}_{\mathrm{n}}^{\delta}$ is chosen as iterative value for calculating $\mathrm{x}_{\mathrm{n}+1}^{\delta}$. When stopping iterative number is determined by discrepancy principle, we have

$$
\mathrm{x}_{\mathrm{n}(\delta)}^{\delta} \rightarrow \mathrm{x}^{+} \quad \delta \rightarrow 0 .
$$

Proof. The condition (ii) assures theorem holds, the condition (i), (iii) and (iv) assure theorem holds and the condition (iii) assures $\mathrm{r}^{\delta} \leq \mathrm{r}(\alpha)$. Therefore, we can always get

$$
\mathrm{x}_{\mathrm{n}}^{\delta} \in \mathrm{K}_{\mathrm{r}}^{\delta}\left(\mathrm{x}_{\mathrm{n}+1}^{\delta}\right) \in \mathrm{K}_{\mathrm{r}(\alpha)}\left(\mathrm{x}_{\mathrm{n}+1}^{\delta}\right)
$$

with $\mathrm{x}_{\mathrm{n}+1}^{\delta} \in \mathrm{K}_{\mathrm{r}}^{\delta}\left(\mathrm{x}_{\mathrm{n}}^{\delta}\right)$. The following conclusion can be gotten by theorem and induction.

As mentioned earlier, the IIGRA algorithm is a combination of the steepest descent method for the minimization of the modified Tikhonov functional and an optimization routine for finding a regularization parameter $\alpha$ such that Morozov's discrepancy principle[1] holds. The algorithm is defined as follows:

Algorithm 13 IIGRA

step $(1)$ choose $x_{0}^{\delta}$ and $\alpha$ satisfied theorem 7 , give $\tau>1$ and $\varepsilon>0$, let $\mathrm{n}=0$; 
$\operatorname{step}(2)$ let $\mathrm{k}=0, \mathrm{x}_{\mathrm{n}, \mathrm{k}}^{\delta}=\mathrm{x}_{\mathrm{n}}^{\delta}$;

step(3) calculate $\nabla \mathrm{J}_{\alpha}\left(\mathrm{x}_{\mathrm{n}+1, \mathrm{k}}^{\delta}, \mathrm{x}_{\mathrm{n}}^{\delta}\right)$;

$\operatorname{step}(4)$ if $\left\|\nabla \mathrm{J}_{\alpha}\left(\mathrm{x}_{\mathrm{n}+1, \mathrm{k}}^{\delta}, \mathrm{x}_{\mathrm{n}}^{\delta}\right)\right\| \leq \varepsilon$, goto $\operatorname{step}(6)$;

step(5) choose $\beta_{\mathrm{k}}$ by theorem 6 and calculate

$$
\mathrm{x}_{\mathrm{n}, \mathrm{k}+1}^{\delta}=\mathrm{x}_{\mathrm{n}, \mathrm{k}}^{\delta}+\beta_{\mathrm{k}} \nabla \mathrm{J}_{\alpha}\left(\mathrm{x}_{\mathrm{n}, \mathrm{k}}^{\delta}, \mathrm{x}_{\mathrm{n}}^{\delta}\right), \mathrm{k}=\mathrm{k}+1 \text {, goto }
$$

$\operatorname{step}(3)$;

$\operatorname{step}(6) \mathrm{x}_{\mathrm{n}+1}^{\delta}=\mathrm{x}_{\mathrm{n}, \mathrm{k}}^{\delta}$;

$\operatorname{step}(7)$ if $\left\|\mathrm{y}^{\delta}-\mathrm{F}\left(\mathrm{x}_{\mathrm{n}+1}^{\delta}\right)\right\| \leq \tau \delta$, stop, otherwise let $\mathrm{n}=\mathrm{n}+1$ and goto $\operatorname{step}(2)$.

\section{TWO NUMERICAL EXPERIMENTS}

Within this section we will give two practical relevant examples that meet the requirements of the IIGRA algorithm.

Example 1: nonlinear Hammerstein integral equation

$$
\begin{aligned}
& \mathrm{F}(\mathrm{x}) \mathrm{s}:=\int_{0}^{\mathrm{t}} \varphi(\mathrm{x}(\mathrm{s})) \mathrm{ds}=\mathrm{y}^{\delta} \\
& \mathrm{D}(\mathrm{F}):=\mathrm{H}^{1}[0,1] \rightarrow \mathrm{L}^{2}[0,1]
\end{aligned}
$$

where assumption 1 are satisfied[2].

The first Fréchet derivate and transposition of operator $\mathrm{F}$ have the following form:

$$
\begin{aligned}
& \left(F^{\prime}(x) h\right)(t)=\int_{0}^{t} \varphi^{\prime}(x(s)) h(s) d s, \\
& \left(F^{\prime}(x)^{*} h\right)(t)=B^{-1}\left[\varphi^{\prime}(x(s)) \int_{s}^{1} h(t) d t .\right]
\end{aligned}
$$

where

$$
\begin{aligned}
& \left(\mathrm{B}^{-1} \mathrm{z}\right)(\mathrm{s})=\int_{0}^{1} \mathrm{k}(\mathrm{s}, \mathrm{t}) \mathrm{z}(\mathrm{t}) \mathrm{dt} . \\
& \mathrm{k}(\mathrm{s}, \mathrm{t}):= \begin{cases}\frac{\cosh (1-\mathrm{s}) \cosh (\mathrm{t})}{\sinh (1)} & \mathrm{t} \leq \mathrm{s} \\
\frac{\cosh (1-\mathrm{t}) \cosh (\mathrm{s})}{\sinh (1)} & \mathrm{t}>\mathrm{s}\end{cases}
\end{aligned}
$$

Therefore, the iterative scheme of IIGRA is:

$\mathrm{B}^{-1}\left[\varphi^{\prime}(\mathrm{x}(\mathrm{s})) \int_{\mathrm{s}}^{1} \mathrm{~h}(\mathrm{t}) \mathrm{dt}.\right]$

$=\int_{0}^{1} \mathrm{k}(\mathrm{s}, \mathrm{t}) \varphi^{\prime}(\mathrm{x}(\mathrm{t}))\left[\int_{\mathrm{t}}^{1} \mathrm{y}^{\delta}(\xi) \mathrm{d} \xi-\int_{\mathrm{t}}^{1} \int_{0}^{\xi} \varphi(\mathrm{x}(\eta)) \mathrm{d} \eta \mathrm{d} \xi\right] \mathrm{dt}$.

Set $\varphi(x)=e^{x}$, then true solution $x^{+}=\sin (t)$. We use IIGRA algorithm solve example 1 in different error level $\delta$ with iteration initial value $\mathrm{x}_{0}(\mathrm{t})=\mathrm{t}, \tau=1.1, \varepsilon=1 \mathrm{e}-5, \alpha=50$.

Table I shows the calculation results of example 1 where $\mathrm{e}_{0}=\left\|\mathrm{a}_{0}^{\delta}-\mathrm{a}^{+}\right\|_{\mathrm{L}}^{2}, \quad \mathrm{e}_{\mathrm{n}}=\left\|\mathrm{a}_{\mathrm{n}}^{\delta} \quad-\mathrm{a}^{+}\right\|_{\mathrm{L}}^{2}$ and $\mathrm{n}(\delta)$ is iteration numbers. We can see that absolute error $e_{n}$ decreases with $\delta$ minishing. This is consistent of the conclusion in theorem 11. Fig.1-Fig.4 give the figures of $\mathrm{x}_{0}, \mathrm{x}^{+}$and $\mathrm{x}_{\mathrm{n}}^{\delta}$ in different $\delta$.

TABLE I.

The NumericAl Results OF EXAMPLE 1 In DifFERENT $\delta$

\begin{tabular}{|c|c|c|c|c|}
\hline$\delta$ & $\mathrm{n}(\delta)$ & $\mathrm{e}_{0}$ & $\mathrm{e}_{\mathrm{n}}$ & $\mathrm{e}_{\mathrm{n} /} \mathrm{e}_{0}$ \\
\hline $1 \mathrm{e}-2$ & 93 & $6.059 \mathrm{e}-2$ & $1.808 \mathrm{e}-2$ & 0.289 \\
\hline $5 \mathrm{e}-3$ & 118 & $6.059 \mathrm{e}-2$ & $1.131 \mathrm{e}-2$ & 0.187 \\
\hline $1 \mathrm{e}-3$ & 233 & $6.059 \mathrm{e}-2$ & $3.720 \mathrm{e}-3$ & $6.159 \mathrm{e}-2$ \\
\hline $5 \mathrm{e}-4$ & 78 & $6.059 \mathrm{e}-2$ & $3.281 \mathrm{e}-3$ & $5.415 \mathrm{e}-2$ \\
\hline $1 \mathrm{e}-5$ & 72 & $6.059 \mathrm{e}-2$ & $2.001 \mathrm{e}-3$ & $3.303 \mathrm{e}-2$ \\
\hline $5 \mathrm{e}-6$ & 84 & $6.059 \mathrm{e}-2$ & $9.804 \mathrm{e}-4$ & $1.618 \mathrm{e}-2$ \\
\hline $1 \mathrm{e}-7$ & 84 & $6.059 \mathrm{e}-2$ & $1.205 \mathrm{e}-4$ & $1.989 \mathrm{e}-3$ \\
\hline $5 \mathrm{e}-8$ & 84 & $6.059 \mathrm{e}-2$ & $5.356 \mathrm{e}-5$ & $8.840 \mathrm{e}-4$ \\
\hline
\end{tabular}

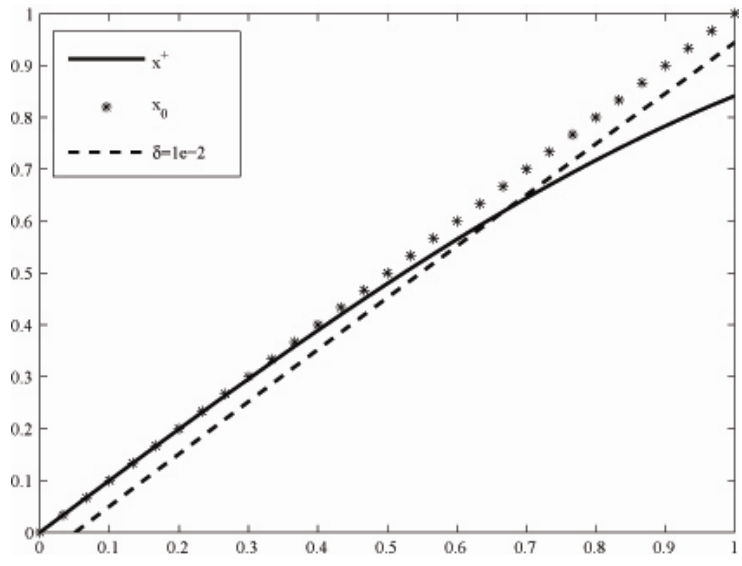

Figure 1. The numerical effect figure with $\delta=1 \mathrm{e}-2$.

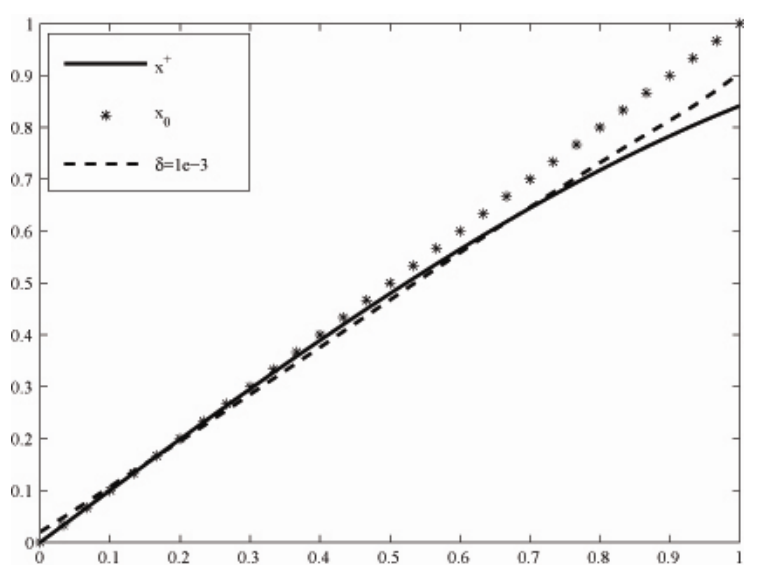

Figure 2. The numerical effect figure with $\delta=1 \mathrm{e}-3$.

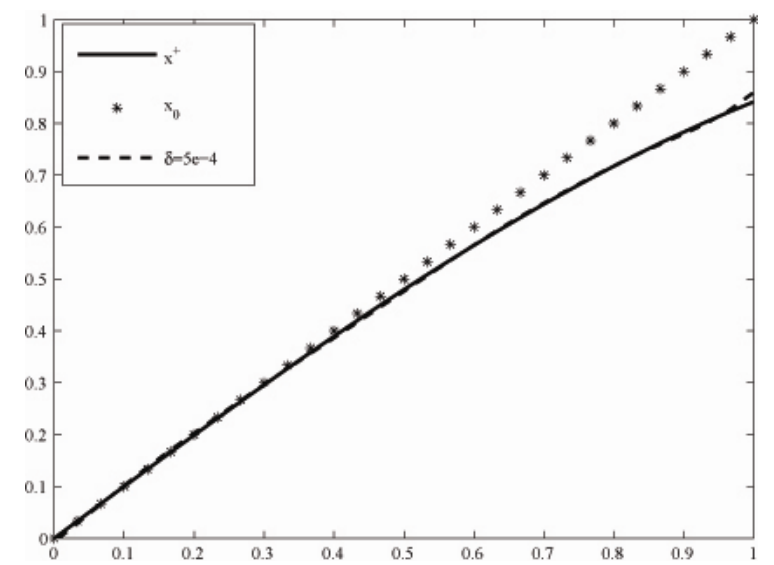

Figure 3. The numerical effect figure with $\delta=5 \mathrm{e}-4$. 


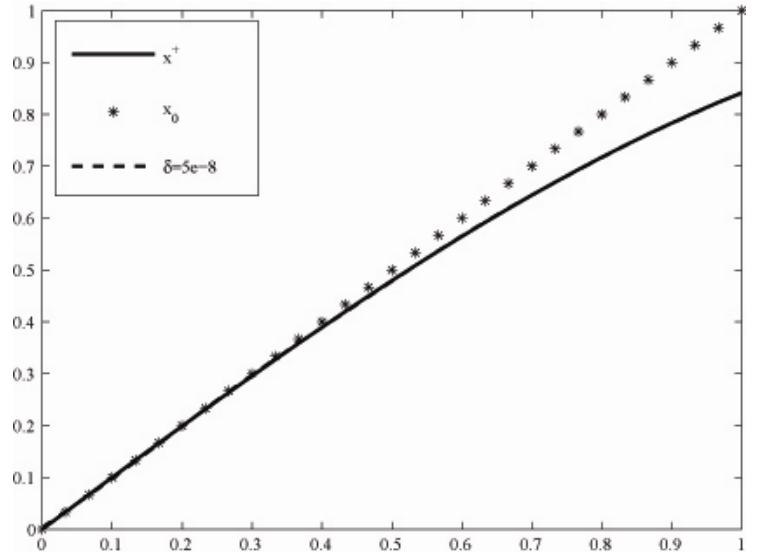

Figure 4. The numerical effect figure with $\delta=5 \mathrm{e}-8$.

Example 2: parameter identification $\mathrm{a}(\mathrm{x})$ in $\mathrm{BVP}$

$$
\left\{\begin{array}{l}
-\left(\mathrm{au}_{\mathrm{x}}\right)_{\mathrm{x}}=-\mathrm{e}^{\mathrm{x}} \\
\mathrm{u}(0)=1 \\
\mathrm{u}(1)=\mathrm{e}
\end{array}\right.
$$

where $\mathrm{a}(\mathrm{x}) \in \mathrm{H}^{1}[0,1]$ and assumption 1 are satisfied[5]. Indeed, example 2 defines a nonlinear operator $\mathrm{F}$ :

$$
\begin{aligned}
& \mathrm{F}(\mathrm{a})=\mathrm{u}(\mathrm{a}) \\
& \mathrm{D}(\mathrm{F})=\left\{\mathrm{H}^{1}[0,1] \mid \mathrm{a} \geq \mathrm{a}^{*}>0\right\} .
\end{aligned}
$$

TABLE II.

The Numerical RESUlts OF EXAMPLE 2 In DifFERENT $\mathrm{a}_{0}^{\delta}$

\begin{tabular}{|c|c|c|c|c|}
\hline $\mathrm{a}_{0}^{\delta}$ & $\mathrm{n}(\delta)$ & $\mathrm{e}_{0}$ & $\mathrm{e}_{\mathrm{n}}$ & $\mathrm{e}_{\mathrm{n} /} \mathrm{e}_{0}$ \\
\hline 0.7 & 67 & 0.298 & 0.138 & 0.463 \\
\hline 3 & 79 & 1.98 & 1.243 & 0.628 \\
\hline 7 & 332 & 5.953 & 3.992 & 0.671 \\
\hline $1+0.05 \sin (10 \pi \mathrm{t})$ & 44 & $3.532 \mathrm{e}-2$ & $5.421 \mathrm{e}-3$ & 0.153 \\
\hline $1+0.1 \sin (10 \pi \mathrm{t})$ & 49 & $7.067 \mathrm{e}-2$ & $8.564 \mathrm{e}-3$ & 0.121 \\
\hline $1+0.3 \sin (10 \pi \mathrm{t})$ & 58 & 0.212 & $8.772 \mathrm{e}-2$ & 0.413 \\
\hline
\end{tabular}

\section{CONCLUSION}

Under some not very strict assumptions, we overcome the difficulty of local convergence for nonlinear ill-posed operator in numerical implementation. Results of examples show its convergence and effectivity. In the next work, we will focus on weakening the restrictions on the property of nonlinear operator.

\section{REFERENCES}

[1] A. N. Tikhonov, A. S. Leonov and A. G. Yagola, Nonlinear Ill-posed Problems. Chapman and Hall: London, 1998.

[2] H. W. Engl, M. Hanke and A. Neubauer, Regularization of Inverse Problems. Kluwer: Dordrecht, 1996.

[3] L. Jianjun, H. Guoqiang and K.Chuangang, "Nonlinear Implicit Iterative Method for Solving Nonlinear Ill-posed Problems,". Appl. Math. Mech. -Engl. Ed. Shanghai University Press. ShangHai, vol. 9, pp. 1183-1192, April 2009.

[4] L. Jianjun, Nonlinear implicit iterative method and regularization GMRES method for solving nonlinear illposed problems[D].(in Chinese) Shanghai University, 2008.

[5] M. Hanke, "A Regularizing Levenberg-Marquardt Scheme with Applications to Inverse Ground-water Filtration Problem,". Inverse Problems. IOP Publlishing. Bristol, vol. 13, pp. 79-95, 1997

\section{ACKNOWLEDGMENT}

The authors wish to thank Qing Miao, Bo Xu and the anonymous referees for their helpful remarks.

Jianjun liu was born in Aug. 6, 1980, China. Science PhD degree was earned from ShangHai University in 2008. The research field is numerical algorithms for ill-posed problems. 